Stochastics and Statistics

\title{
Expected gain-loss pricing and hedging of contingent claims in incomplete markets by linear programming is
}

\author{
Mustafa Ç. Pınar ${ }^{\mathrm{a}, *}$, Aslıhan Salih ${ }^{\mathrm{b}}$, Ahmet Camci ${ }^{\mathrm{a}}$ \\ ${ }^{a}$ Department of Industrial Engineering, Bilkent University, 06800 Ankara, Turkey \\ ${ }^{\mathrm{b}}$ Department of Management, Bilkent University, 06800 Ankara, Turkey
}

\section{A R T I C L E I N F O}

\section{Article history:}

Received 30 May 2007

Accepted 24 February 2009

Available online 3 March 2009

\section{Keywords:}

Contingent claim

Pricing

Hedging

Martingales

Stochastic linear programming

Transaction costs

\begin{abstract}
A B S T R A C T
We analyze the problem of pricing and hedging contingent claims in the multi-period, discrete time, discrete state case using the concept of a " $\lambda$ gain-loss ratio opportunity". Pricing results somewhat different from, but reminiscent of, the arbitrage pricing theorems of mathematical finance are obtained. Our analysis provides tighter price bounds on the contingent claim in an incomplete market, which may converge to a unique price for a specific value of a gain-loss preference parameter imposed by the market while the hedging policies may be different for different sides of the same trade. The results are obtained in the simpler framework of stochastic linear programming in a multi-period setting, and have the appealing feature of being very simple to derive and to articulate even for the non-specialist. They also extend to markets with transaction costs.
\end{abstract}

(c) 2009 Elsevier B.V. All rights reserved.

\section{Introduction}

An important class of pricing theories in financial economics are derived under no-arbitrage conditions. In complete markets, these theories yield unique prices without any assumptions about individual investor's preferences. In other words, the pricing of assets relies on the availability and the liquidity of traded assets that span the full set of possible future states. Ross [26,27] proves that the no-arbitrage condition is equivalent to the existence of a linear pricing rule and positive state prices that correctly value all assets. This linear pricing rule is the risk neutral probability measure in the Cox-Ross option pricing model, for example Harrison and Kreps [13] showed that the linear pricing operator is an expectation taken with respect to a martingale measure. However, when markets are incomplete state prices and claim prices are not unique. Since markets are almost never complete due to market imperfections as discussed in Carr et al. [5], and characterizing all possible future states of economy is impossible, alternative incomplete pricing theories have been developed.

In an incomplete financial market with no-arbitrage opportunities, a noticeable feature of the set of risk neutral measures is that the value of the cheapest portfolio to dominate the pay-off at maturity of a contingent claim coincides with the maximum expected value of the (discounted) pay-off of the claim with respect to this set. This value, which may be called the writer's price, allows the writer to assemble a hedge portfolio that achieves a value at least as large as the pay-off to the claim holder at the maturity date of the claim in all non-negligible events. The writer's price is the natural price to be asked by the writer (seller) of a contingent claim and, together with the bid price obtained by considering the analogous problem from the point of view of the buyer, forms an interval which is sometimes called the "no-arbitrage price interval" for the claim in question.

A writer may nevertheless be induced for various reasons to settle for less than the above price to sell a claim with pay-off $F_{T}$; see e.g., Chapters 7 and 8 of [10] for a discussion and examples showing that the writer's price may be too high. In such a case, he/she will not be able to set up a portfolio dominating the claim pay-off almost surely, which implies that he/she will face a positive probability of "falling short", i.e., his/her hedge portfolio will take values $V_{T}$ smaller than those of the claim on a non-negligible event. Thus, the writer will need to choose his/her hedge portfolio (and selling price) according to some optimality criterion to be decided. The gain-loss pricing criterion of the present paper inspired by the gain-loss ratio criterion of Bernardo and Ledoit [2] suggests to choose the portfolio which gives the best value of the difference of expected positive final positions and a parameter $\lambda$ (greater than one) times the expected negative final positions,

\footnotetext{
This research is partially supported by TUBITAK Grant 107K250.

* Corresponding author.

E-mail addresses: mustafap@bilkent.edu.tr (M.Ç. Pınar), asalih@bilkent.edu.tr (A. Salih), camci@bilkent.edu.tr (A. Camcı).
} 
$\mathbb{E}\left[\left(V_{T}-F_{T}\right)_{+}\right]-\lambda \mathbb{E}\left[\left(V_{T}-F_{T}\right)_{-}\right]$, aimed at weighting "losses" more than "gains". This criterion gives rise to a new concept different from the ordinary arbitrage, the " $\lambda$ gain-loss ratio opportunity", i.e., a portfolio which can be set up at no cost but yields a positive value for the difference between gains and " $\lambda$-losses". In this paper, we show that the price processes in a multiple period, discrete time, finite state financial market do not admit a $\lambda$ gain-loss ratio opportunity if and only if there exists an equivalent martingale measure with an additional restriction. As for the maximum and minimum no-arbitrage prices, we determine the maximum and minimum prices which do not introduce $\lambda$ gain-loss opportunities in the market. Thus, a new price interval (the " $\lambda$ gain-loss price interval") is determined, generally contained in the no-arbitrage interval (thus more significant from an economical point of view since it is more restrictive). These prices converge to the no-arbitrage bounds in the limit as the gain-loss preference parameter goes to infinity (and hence, the investor essentially looks for an arbitrage). On the other extreme, our results show that the market may actually arrive at a consensus about the pricing rule, i.e., as the gain-loss preference parameter goes down to the smallest value not allowing a $\lambda$ gain-loss ratio opportunity, the writer and buyer's no- $\lambda$ gain-loss ratio opportunity prices of a contingent claim may converge to a single value, hence potentially providing a unique price for the contingent claim in an incomplete market. However, in the incomplete market setting, the same pricing rule leads to different hedging policies for different sides of the same trade. This is an important finding as it will result in different demand and supply schemes for the replicating assets. An attractive feature of our results is that all derivations and computations are carried out using linear programming models derived from simple stochastic programming formulations, which offer a propitious framework for adding additional variables and constraints into the models as well as possibility of efficient numerical processing; see the book [3] for a thorough introduction to stochastic programming.

Our concept of $\lambda$ gain-loss ratio opportunity is akin to the notion of a good deal that was developed in a series of papers by various authors $[6,8,18,28]$. For example in Cochrane and Saa-Requejo [8], the absence of arbitrage is replaced by the concept of a good deal, defined as an investment with a high Sharpe ratio. While they do not use the term "good-deal", Bernardo and Ledoit [2] replace the high Sharpe ratio by the gain-loss ratio. These earlier studies are carried out using duality theory in infinite dimensional spaces in [6,18,28], usually in singleperiod models. Working with single-period models is not necessarily a limitation since dynamic models with a fixed terminal date can be viewed as one-period models with investment choices taking values in suitable spaces. Recent work on risk measures and portfolio optimization, e.g. [10], adopts this approach to formulate single-period problems using function spaces rich enough to be extended to multi-period or continuous time markets; see Section 8 of Staum [28] for a discussion. In this regard, the contribution of the present paper is to make explicit which consequences can general single-period results have when applied to multi-period discrete space markets.

We note that a second class of pricing theories relies on the expected utility framework which posits that if preferences satisfy a number of axioms, then they can be represented by an expected utility function. This framework requires the specification of investor preferences through usually non-linear utility functions; see Chapter 1 of [16]. This model equates the price of a claim to the expectation of the product of the future pay-off and the marginal rate of substitution of the representative investor; see e.g. [7,15,20] for related recent work. Recent papers by Cochrane and Saa-Requejo [8], Bernardo and Ledoit [2], Carr et al. [5], Roorda et al. [25] and Kallsen [20] unify these two classes of pricing theories and value options in an incomplete market setting. In the present paper, we work with linear programming models, and avoid the non-linearities encountered with utility functions. Our notion of gain-loss ratio opportunity is also related to prospect theory of Kahneman and Tversky [19] proposed as an alternative to expected utility framework. In prospect theory, it is presumed based on experimental evidence that gains and losses have asymmetric effects on the agents' welfare where welfare, or utility, is defined not over total wealth but over gains and losses; see Grüne and Semmler [12] and Barberis et al. [1] for details on the use of the gain-loss function as a central part of welfare functions in asset pricing.

The organization of the paper is as follows. In Section 2, we review the stochastic process governing the asset prices and we lay out the basics of our analysis. Section 3 gives a characterization of the absence of a $\lambda$ gain-loss ratio opportunity in terms of martingale measures. We consider a related problem in Section 4 where the investor in search of a $\lambda$ gain-loss ratio opportunity would also like to find the $\lambda$ gainloss ratio opportunity with the limiting value of the parameter $\lambda$. Here we re-obtain a duality result which turns out be essentially the duality result of Bernardo and Ledoit in a multi-period but finite probability state space setting. In Section 5 we analyze the pricing problems of writers and buyers of contingent claims under the $\lambda$ gain-loss ratio opportunity viewpoint. We extend the results of the paper to markets with transaction costs in Section 6. We use simple numerical examples to illustrate our results.

\section{The stochastic scenario tree, arbitrage and martingales}

Throughout this paper we follow the general probabilistic setting of $[21,29]$ where we model the behavior of the stock market by assuming that security prices and other payments are discrete random variables supported on a finite probability space $(\Omega, \mathscr{F}, P)$ whose atoms $\omega$ are sequences of real-valued vectors (asset values) over the discrete time periods $t=0,1, \ldots, T$. For a general reference on mathematical finance in discrete time, finite state markets the reader is referred to Pliska [23]. A recent reference treating option pricing from the optimization point of view in discrete time, finite state markets is [11]. We assume the market evolves as a discrete, non-recombinant scenario tree in which the partition of probability atoms $\omega \in \Omega$ generated by matching path histories up to time $t$ corresponds one-to-one with nodes $n \in \mathscr{N}_{t}$ at level $t$ in the tree. The set $\mathscr{N}_{0}$ consists of the root node $n=0$, and the leaf nodes $n \in \mathscr{N}_{T}$ correspond one-to-one with the probability atoms $\omega \in \Omega$. In the scenario tree, every node $n \in \mathscr{N}_{t}$ for $t=1, \ldots, T$ has a unique parent denoted $\pi(n) \in \mathscr{N}_{t-1}$, and every node $n \in \mathscr{N}_{t}, t=0,1, \ldots, T-1$ has a non-empty set of child nodes $\mathscr{S}(n) \subset \mathscr{N}_{t+1}$. The set of all ascendant nodes and all descendant nodes of a node $n$ are denoted $A(n)$, and $D(n)$, respectively, in both cases including node $n$ itself. We denote the set of all nodes in the tree by $\mathscr{N}$. The probability distribution $P$ is obtained by attaching positive weights $p_{n}$ to each leaf node $n \in \mathscr{N}_{T}$ so that $\sum_{n \in \mathscr{N}_{T}} p_{n}=1$. For each non-terminal (intermediate level) node in the tree we have, recursively,

$$
p_{n}=\sum_{m \in \mathscr{S}(n)} p_{m}, \quad \forall n \in \mathscr{N}_{t}, t=T-1, \ldots, 0 .
$$

Hence, each intermediate node has a probability mass equal to the combined mass of the paths passing through it. The ratios $p_{m} / p_{n}, m \in \mathscr{S}(n)$ are the conditional probabilities that the child node $m$ is visited given that the parent node $n=\pi(m)$ has been visited. This setting is chosen as it accommodates multi-period pricing for future different states and time periods at the same time, employing 
realization paths in the valuation process. It is a framework that allows to address the valuation problem with incomplete markets and heterogeneous beliefs which are very stringent assumptions in the classical valuation theory. In this respect, it improves our understanding of valuation in a simple, yet complete fashion.

A random variable $X$ is a real-valued function defined on $\Omega$. It can be lifted to the nodes of a partition $\mathcal{N}_{t}$ of $\Omega$ if each level set $\left\{X^{-1}(a): a \in \mathbb{R}\right\}$ is either the empty set or is a finite union of elements of the partition. In other words, $X$ can be lifted to $\mathscr{N}_{t}$ if it can be assigned a value on each node of $\mathscr{N}_{t}$ that is consistent with its definition on $\Omega$ [21]. This kind of random variable is said to be measurable with respect to the information contained in the nodes of $\mathscr{N}_{t}$. A stochastic process $\left\{X_{t}\right\}$ is a time-indexed collection of random variables such that each $X_{t}$ is measurable with respect to $\mathscr{N}_{t}$. The expected value of $X_{t}$ is uniquely defined by the sum

$$
\mathbb{E}^{P}\left[X_{t}\right]:=\sum_{n \in \mathcal{N}_{t}} p_{n} X_{n}
$$

The conditional expectation of $X_{t+1}$ on $\mathscr{N}_{t}$ is a random variable taking values over the nodes $n \in \mathscr{N}_{t}$, given by the expression

$$
\mathbb{E}^{P}\left[X_{t+1} \mid \mathscr{N}_{t}\right]:=\sum_{m \in \mathscr{S}(n)} \frac{p_{m}}{p_{n}} X_{m}
$$

Under the light of the above definitions, the market consists of $J+1$ tradable securities indexed by $j=0,1, \ldots, J$ with prices at node $n$ given by the vector $S_{n}=\left(S_{n}^{0}, S_{n}^{1}, \ldots, S_{n}^{J}\right)$. We assume as in [21] that the security indexed by 0 has strictly positive prices at each node of the scenario tree. Furthermore, the price of the security indexed by 0 grows by a given factor in each time period. This asset corresponds to the risk-free asset in the classical valuation framework. Choosing this security as the numéraire, and using the discount factors $\beta_{n}=1 / S_{n}^{0}$ we define $Z_{n}^{j}=\beta_{n} S_{n}^{j}$ for $j=0,1, \ldots, J$ and $n \in \mathscr{N}$, the security prices discounted with respect to the numéraire. Note that $Z_{n}^{0}=1$ for all nodes $n \in \mathscr{N}$, and $\beta_{n}$ is a constant, equal to, $\beta_{t}$, for all $n \in \mathscr{N}_{T}$, for a fixed $t \in[0, \ldots, T]$.

The amount of security $j$ held by the investor in state (node) $n \in \mathscr{N}_{t}$ is denoted $\theta_{n}^{j}$. Therefore, to each state $n \in \mathscr{N}_{t}$ is associated a vector $\theta_{n} \in \mathbb{R}^{J+1}$. We refer to the collection of vectors $\theta_{n}$ for all $n \in \mathscr{N}$ as $\Theta$. The value of the portfolio at state $n$ (discounted with respect to the numéraire) is

$$
Z_{n} \cdot \theta_{n}=\sum_{j=0}^{J} Z_{n}^{j} \theta_{n}^{j} .
$$

We will work with the following definition of arbitrage: an arbitrage is a sequence of portfolio holdings that begins with a zero initial value (note that short sales are allowed), makes self-financing portfolio transactions throughout the planning horizon and achieves a nonnegative terminal value in each state, while in at least one terminal state it achieves a positive value with non-zero probability. The selffinancing transactions condition is expressed as

$$
Z_{n} \cdot \theta_{n}=Z_{n} \cdot \theta_{\pi(n)}, \quad n>0 .
$$

The stochastic programming problem used to seek an arbitrage is the following optimization problem (P1):

$$
\begin{array}{ll}
\max & \sum_{n \in \mathcal{N}_{T}} p_{n} Z_{n} \cdot \theta_{n} \\
\text { s.t. } & Z_{0} \cdot \theta_{0}=0 \\
& Z_{n} \cdot\left(\theta_{n}-\theta_{\pi(n)}\right)=0, \quad \forall n \in \mathscr{N}_{t}, t \geqslant 1 \\
& Z_{n} \cdot \theta_{n} \geqslant 0, \quad \forall n \in \mathscr{N}_{T} .
\end{array}
$$

If there exists an optimal solution (i.e., a sequence of vectors $\theta_{n}$ for all $n \in \mathscr{N}$ ) which achieves a positive optimal value, this solution can be turned into an arbitrage as demonstrated by Harrison and Pliska [14].

We need the following definitions.

Definition 1. If there exists a probability measure $Q=\left\{q_{n}\right\}_{n \in \mathcal{N}_{\mathscr{F}}}$ (extended to intermediate nodes recursively as in (1)) such that

$$
Z_{t}=\mathbb{E}^{Q}\left[Z_{t+1} \mid \mathscr{N}_{t}\right] \quad(t \leqslant T-1)
$$

then the vector process $\left\{Z_{t}\right\}$ is called a vector-valued martingale under $Q$ and $Q$ is called a martingale probability measure for the process. If one has coordinate-wise $Z_{t} \geqslant \mathbb{E}^{Q}\left[Z_{t+1} \mid \mathscr{N}_{t}\right],(t \leqslant T-1)$ (respectively, $Z_{t} \leqslant \mathbb{E}^{Q}\left[Z_{t+1} \mid \mathscr{N}_{t}\right],(t \leqslant T-1)$ ) the process is called a super-martingale (sub-martingale, respectively).

Definition 2. A discrete probability measure $Q=\left\{q_{n}\right\}_{n \in \mathcal{N}_{T}}$ is equivalent to a (discrete) probability measure $P=\left\{p_{n}\right\}_{n \in \mathscr{N}_{T}}$ if $q_{n}>0$ exactly when $p_{n}>0$.

King proved the following theorem (c.f. Theorem 1 of [21]).

Theorem 1. The discrete state stochastic vector process $\left\{Z_{t}\right\}$ is an arbitrage-free market price process if and only if there is at least one probability measure $Q$ equivalent to $P$ under which $\left\{Z_{t}\right\}$ is a martingale.

The above result is the equivalent of Theorem 1 of Harrison and Kreps [13] in our setting.

\section{Gain-loss ratio opportunities and martingales}

In our context a $\lambda$ gain-loss ratio opportunity is defined as follows. For $n \in \mathscr{N}_{T}$ let $Z_{n} \cdot \theta_{n}=x_{n}^{+}-x_{n}^{-}$where $x_{n}^{+}$and $x_{n}^{-}$are non-negative numbers, i.e., we express the final portfolio value at terminal state $n$ as the sum of positive and negative positions $\left(x_{n}^{+}\right.$denotes the gain at node $n$ while $x_{n}^{-}$stands for the loss at node $n$ ). Assume that there exist vectors $\theta_{n}$ for all $n \in \mathscr{N}$ such that 


$$
\begin{aligned}
& Z_{0} \cdot \theta_{0}=0, \\
& Z_{n} \cdot\left(\theta_{n}-\theta_{\pi(n)}\right)=0, \quad \forall n \in \mathscr{N}_{t}, t \geqslant 1
\end{aligned}
$$

and

$$
\mathbb{E}^{P}\left[X^{+}\right]-\lambda \mathbb{E}^{P}\left[X^{-}\right]>0
$$

for $\lambda>1$, where $X^{+}=\left\{x_{n}^{+}\right\}_{n \in \mathcal{N}_{T}}$, and $X^{-}=\left\{x_{n}^{-}\right\}_{n \in \mathcal{N}_{T}}$. This sequence of portfolio holdings is said to yield a $\lambda$ gain-loss ratio opportunity (for a fixed value of $\lambda$ ). This formulation is similar to Bernardo and Ledoit [2] gain-loss ratio, and the Sharpe ratio restriction of Cochrane and SaaRequejo [8]. Yet, it makes the problem easier to tackle within the framework of linear programming. Moreover, the parameter $\lambda$ can be interpreted as the gain-loss preference parameter of the individual investor. As $\lambda$ gets bigger, the individual's aversion to loss is becoming more and more pronounced, since he/she begins to prefer near-arbitrage positions. As $\lambda$ gets closer to 1 , the individual weighs the gains and losses equally. In the limiting case of $\lambda$ being equal to 1 the pricing operator (equivalent martingale measure) is unique if it exists. In fact, the pricing operator may become unique at a value of $\lambda$ larger than one, which is what we expect in a typical pricing problem.

Consider now the perspective of an investor who is content with the existence of a $\lambda$ gain-loss ratio opportunity although an arbitrage opportunity does not exist. Such an investor is interested in the solution of the following stochastic linear programming problem that we refer to as (SP1):

$$
\begin{array}{ll}
\max & \sum_{n \in \mathscr{N}_{T}} p_{n} x_{n}^{+}-\lambda \sum_{n \in \mathscr{N}_{T}} p_{n} x_{n}^{-} \\
\text {s.t. } & Z_{0} \cdot \theta_{0}=0, \\
& Z_{n} \cdot\left(\theta_{n}-\theta_{\pi(n)}\right)=0, \quad \forall n \in \mathscr{N}_{t}, t \geqslant 1, \\
& Z_{n} \cdot \theta_{n}-x_{n}^{+}+x_{n}^{-}=0, \quad \forall n \in \mathscr{N}_{T}, \\
& x_{n}^{+} \geqslant 0, \quad \forall n \in \mathscr{N}_{T}, \\
& x_{n}^{-} \geqslant 0, \quad \forall n \in \mathscr{N}_{T} .
\end{array}
$$

If there exists an optimal solution (i.e., a sequence of vectors $\theta_{n}$ for all $n \in \mathscr{N}$ ) to the above problem that yields a positive optimal value, the solution is said to give rise to a $\lambda$ gain-loss ratio opportunity (the expected positive terminal wealth outweighing $\lambda$ times the expected negative final wealth). If there exists a $\lambda$ gain-loss ratio opportunity in SP1, then SP1 is unbounded. We note that by the fundamental theorem of linear programming, when it is solvable, SP1 has always a basic optimal solution in which no pair $x_{n}^{+}, x_{n}^{-}$, for all $n \in \mathscr{N}_{T}$, can be positive at the same time.

We will say that the discrete state stochastic vector process $\left\{Z_{t}\right\}$ does not admit a $\lambda$ gain-loss ratio opportunity (at a fixed value of $\lambda$ ) if the optimal value of the above stochastic linear program is equal to zero. Clearly, if $\lambda$ tends to infinity we essentially recover King's problem P1. It is a well-accepted phenomenon that every rational investor is ready to lose if the benefits of the gains outweigh the costs of the losses [19]. It is also reasonable to assume that the rational investor will try to limit losses. This type of behavior excluded by the no-arbitrage setting is easily modeled by the Expected Utility approach and in prospect theory. Our formulation allows investors to take reasonable risks without explicitly specifying a complicated utility function while it converges to the no-arbitrage setting in the limit. It is easy to see that an arbitrage opportunity is also a $\lambda$ gain-loss ratio opportunity, and that absence of a $\lambda$ gain-loss ratio opportunity (at any level $\lambda$ ) implies absence of arbitrage. It follows from Theorem 1 that if the market price process does not admit a $\lambda$ gain-loss ratio opportunity then there exists an equivalent measure that makes the price process a martingale.

Definition 3. Given $\lambda>1$ a discrete probability measure $Q=\left\{q_{n}\right\}_{n \in \mathscr{N}_{T}}$ is $\lambda$-compatible to a (discrete) probability measure $P=\left\{p_{n}\right\}_{n \in \mathscr{N}_{T}}$ if it is equivalent to $P$ (Definition 2 ) and satisfies

$$
\max _{n \in \mathscr{N}_{T}} p_{n} / q_{n} \leqslant \lambda \min _{n \in \mathscr{N}_{T}} p_{n} / q_{n}
$$

Theorem 2. The process $\left\{Z_{t}\right\}$ does not admit $\lambda$ gain-loss ratio opportunity (at a fixed level $\lambda>1$ ) if and only if there exists a probability measure $Q \lambda$-compatible to $P$ which makes the discrete vector price process $\left\{Z_{t}\right\}$ a martingale.

Proof. We prove the necessity part first. We begin by forming the dual problem to SP1. Attaching unrestricted-in-sign dual multiplier $y_{0}$ with the first constraint, multipliers $y_{n},(n>0)$ with the self-financing transaction constraints, and finally multipliers $w_{n},\left(n \in \mathscr{N}_{T}\right)$ with the last set of constraints we form the Lagrangian function:

$$
L\left(\Theta, X^{+}, X^{-}, y, w\right)=\sum_{n \in \mathscr{N}_{T}} p_{n} x_{n}^{+}-\lambda \sum_{n \in \mathscr{N}_{T}} p_{n} x_{n}^{-}+y_{0} Z_{0} \cdot \theta_{0}+\sum_{t=1}^{T} \sum_{n \in \mathcal{N}_{t}} y_{n} Z_{n} \cdot\left(\theta_{n}-\theta_{\pi(n)}\right)+\sum_{n \in \mathscr{N}_{T}} w_{n}\left(Z_{n} \cdot \theta_{n}-x_{n}^{+}+x_{n}^{-}\right)
$$

that we maximize over the variables $\Theta, X^{+}$, and $X^{-}$separately. From these separate maximizations we obtain the following:

$$
\begin{aligned}
& y_{0} Z_{0}=\sum_{n \in \mathscr{S}(0)} y_{n} Z_{n}, \\
& y_{m} Z_{m}=\sum_{n \in \mathscr{S}(m)} y_{n} Z_{n}, \quad \forall m \in \mathscr{N}_{t}, 1 \leqslant t \leqslant T-1, \\
& p_{n} \leqslant y_{n} \leqslant \lambda p_{n}, \forall n \in \mathscr{N}_{T},
\end{aligned}
$$

where we got rid of the dual variables $w_{n}$ in the process by observing that maximizations over $\theta_{n},\left(n \in \mathscr{N}_{T}\right)$ yield the equations

$$
\left(w_{n}-y_{n}\right) Z_{n}=0, \quad \forall n \in \mathscr{N}_{T}
$$

and since the first component $Z_{n}^{0}=1$ for all states $n$, we have $y_{n}=w_{n},\left(n \in \mathscr{N}_{T}\right)$. Therefore, we have obtained the dual problem that we refer to SD1 with an identically zero objective function and the constraints given by (3)-(5). 
Now let us observe that problem SP1 is always feasible (the zero portfolio in all states is feasible) and if there is no $\lambda$ gain-loss ratio opportunity, the optimal value is equal to zero. Therefore, by linear programming duality, the dual problem is also solvable (in fact, feasible since the dual is only a feasibility problem). Let us take any feasible solution $y_{n},(n \in \mathscr{N})$ of the dual system given by (3)-(5). Since the first component, $Z_{n}^{0}$ is equal to 1 in each state $n$, we have that

$$
y_{m}=\sum_{n \in \mathscr{S}(m)} y_{n}, \quad \forall m \in \mathscr{N}_{t}, 1 \leqslant t \leqslant T-1 .
$$

Since $y_{n} \geqslant p_{n}$, it follows that $y_{n}$ is a strictly positive process such that the sum of $y_{n}$ over all states $n \in \mathscr{N}_{t}$ in each time period $t$ sums to $y_{0}$. Now, define the process $q_{n}=y_{n} / y_{0}$, for each $n \in \mathscr{N}$. Obviously, this defines a probability measure $Q$ over the leaf (terminal) nodes $n \in \mathscr{N}_{T}$. Furthermore, we can rewrite (4) with the newly defined weights $q_{n}$ as

$$
q_{m} Z_{m}=\sum_{n \in \mathscr{S}(m)} q_{n} Z_{n}, \quad \forall m \in \mathscr{N}_{t}, 1 \leqslant t \leqslant T-1
$$

with $q_{0}=1$, and all $q_{n}>0$. Therefore, by constructing the probability measure $Q$ we have constructed an equivalent measure which makes the price process $\left\{Z_{t}\right\}$ a martingale according to Definition 1. By definition of the measure $q_{n}$, we have using the inequalities (5)

$$
p_{n} \leqslant q_{n} y_{0} \leqslant \lambda p_{n}, \quad \forall n \in \mathscr{N}_{T}
$$

or equivalently,

$$
p_{n} / q_{n} \leqslant y_{0} \leqslant \lambda p_{n} / q_{n}, \quad \forall n \in \mathscr{N}_{T},
$$

which implies that $q_{n}, n \in \mathscr{N}_{T}$ constitute a $\lambda$-compatible martingale measure. This concludes the necessity part.

Suppose $Q$ is a $\lambda$-compatible martingale measure for the price process $\left\{Z_{t}\right\}$. Therefore, we have

$$
q_{m} Z_{m}=\sum_{n \in \mathscr{S}(m)} q_{n} Z_{n}, \quad \forall m \in \mathscr{N}_{t}, 1 \leqslant t \leqslant T-1,
$$

with $q_{0}=1$, and all $q_{n}>0$, while the condition $\max _{n \in \mathcal{N}_{T}} p_{n} / q_{n} \leqslant \lambda \min _{n \in \mathcal{N}_{T}} p_{n} / q_{n}$ holds. If the previous inequality holds as an equality, choose the right-hand (or, the left-hand) of the inequality as a factor $y_{0}$ and set $y_{n}=q_{n} y_{0}$ for all $n \in \Omega$. If the inequality is not tight, any value $y_{0}$ in the interval $\left[\max _{n \in \mathcal{N}_{T}} p_{n} / q_{n}, \lambda \min _{n \in \mathcal{N}_{T}} p_{n} / q_{n}\right.$ ] will do. It is easily verified that $y_{n}, n \in \mathscr{N}$ so defined satisfy the constraints of the dual problem SD1. Since the dual problem is feasible, the primal SP1 is bounded above (in fact, its optimal value is zero) and no $\lambda$ gain-loss ratio opportunity exists in the system.

As a first remark, we can immediately make a statement equivalent to Theorem 2: The price process (or the market) does not have a $\lambda$ gain-loss ratio opportunity (at fixed level $\lambda$ ) if and only if there exists an equivalent measure $Q$ to $P$ such that:

$$
\frac{\max _{n \in \mathcal{N}_{T}} p_{n} / q_{n}}{\min _{n \in \mathcal{N}_{T}} p_{n} / q_{n}} \leqslant \lambda
$$

or, equivalently

$$
\frac{\max _{n \in \mathscr{N}_{T}} q_{n} / p_{n}}{\min _{n \in \mathcal{N}_{T}} q_{n} / p_{n}} \leqslant \lambda
$$

or,

$$
\frac{\max _{\omega} \frac{d Q}{d P}(\omega)}{\min _{\omega} \frac{d Q}{d P}(\omega)} \leqslant \lambda
$$

using the Radon-Nikodym derivative, and that $Q$ makes the price process a martingale. Clearly, posing the condition as such introduces a non-linear system of inequalities, whereas our equivalent dual problem SD1 is a linear programming problem. After preparing this manuscript we noticed that a similar observation for single-period problems was made in a technical note [22] although the language and notation of this reference is very different from ours.

As a second remark, we note that if we allow $\lambda$ to tend to infinity we find ourselves in King's framework at which point Theorem 1 is valid. Therefore, this theorem is obtained as a special case of Theorem 2.

Example 1. Let us now consider a simple single-period numerical example. Let us assume for simplicity that the market consists of a riskless asset with zero growth rate, and of a stock. The stock price evolves according a trinomial tree as follows. Assume the riskless asset has price equal to one throughout. At time $t=0$, the stock price is 10 . Hence $Z_{0}=\left(\begin{array}{ll}1 & 10\end{array}\right)^{T}$. At the time $t=1$, the stock price can take the values $20,15,7.5$ with equal probability. Therefore, at node 1 one has $Z_{1}=\left(\begin{array}{ll}1 & 20\end{array}\right)^{T}$; at node $2 Z_{2}=\left(\begin{array}{lll}1 & 15\end{array}\right)^{T}$ and finally at node 3 $Z_{3}=\left(\begin{array}{ll}1 & 7.5\end{array}\right)^{T}$. In other words, all $\beta$ factors are equal to one. It is easy to see that the market described above is arbitrage free because we can show the existence of an equivalent martingale measure, e.g., $q_{1}=q_{2}=1 / 8$ and $q_{3}=3 / 4$. Now, setting up and solving the problems SP1 and/or SD1, we observe that for all values of $\lambda \geqslant 6$, no $\lambda$ gain-loss ratio opportunity exists in the market. However, for values of $\lambda$ strictly between one and six, the primal problem SP1 is unbounded and the dual problem SD1 is infeasible. Therefore, $\lambda$ gain-loss ratio opportunities exist.

As $\lambda$ gets smaller, eventually the feasible set of the dual problem reduces to a singleton, at which point an interesting pricing result is observed as we shall see in Section 5. First, we investigate the problem of finding the smallest $\lambda$ not allowing $\lambda$ gain-loss ratio opportunities in the next section. 


\section{Seeking out the highest possible $\lambda$ in a gain-loss ratio opportunity framework}

We have assumed thus far that the parameter $\lambda$ was decided by the agent (writer or buyer) before the solution of the stochastic linear programs of the previous section. However, once a $\lambda$ gain-loss ratio opportunity is found at a certain level of $\lambda$ it is legitimate to ask whether $\lambda$ gain-loss ratio opportunities at higher levels of $\lambda$ continue to exist. In fact, it is natural to wonder how far up one can push $\lambda$ before $\lambda$ gain-loss ratio opportunities cease to exist. Therefore, it is relevant, while seeking $\lambda$ gain-loss ratio opportunities, to consider the following optimization problem LamP1:

$$
\begin{array}{ll}
\text { sup } & \lambda \\
\text { s.t. } & \sum_{n \in \mathscr{N}_{T}} p_{n} x_{n}^{+}-\lambda \sum_{n \in \mathscr{N}_{T}} p_{n} x_{n}^{-}>0, \\
& Z_{0} \cdot \theta_{0}=0, \\
& Z_{n} \cdot\left(\theta_{n}-\theta_{\pi(n)}\right)=0, \quad \forall n \in \mathscr{N}_{t}, \quad t \geqslant 1, \\
& Z_{n} \cdot \theta_{n}-x_{n}^{+}+x_{n}^{-}=0, \quad \forall n \in \mathscr{N}_{T}, \\
& x_{n}^{+} \geqslant 0, \quad \forall n \in \mathscr{N}_{T}, \\
& x_{n}^{-} \geqslant 0, \quad \forall n \in \mathscr{N}_{T} .
\end{array}
$$

Notice that problem LamP1 is a non-convex optimization problem, and as such is potentially very hard. However, it can be posed in a form suitable for numerical processing as we claim by the next proposition (see Appendix for the proof).

Proposition 1. LamP1 is equivalent to the following problem LamPr under the assumption that a $\lambda$ gain-loss ratio opportunity exists

$$
\begin{array}{ll}
\sup & \sum_{n \in \mathcal{N}_{T}} p_{n} x_{n}^{+} \\
& \sum_{n \in \mathcal{N}_{T}} p_{n} x_{n}^{-} \\
\text {s.t. } & Z_{0} \cdot \theta_{0}=0, \\
& Z_{n} \cdot\left(\theta_{n}-\theta_{\pi(n)}\right)=0, \quad \forall n \in \mathscr{N}_{t}, \quad t \geqslant 1, \\
& Z_{n} \cdot \theta_{n}-x_{n}^{+}+x_{n}^{-}=0, \quad \forall n \in \mathscr{N}_{T}, \\
& x_{n}^{+} \geqslant 0, \quad \forall n \in \mathscr{N}_{T}, \\
& x_{n}^{-} \geqslant 0, \quad \forall n \in \mathscr{N}_{T} .
\end{array}
$$

Notice that as a result of the homogeneity of the equalities and inequalities defining the constraints of problem LamPr, if $\Theta, X^{+}, X^{-}$is feasible for LamPr, then so is $\kappa\left(\Theta, X^{+}, X^{-}\right)$for any $\kappa>0$, and the objective function value is constant along such rays.

Assumption 1. The price process $\left\{Z_{t}\right\}$ is arbitrage free, i.e., there does not exist feasible $\Theta, X^{+}, X^{-}$with $\mathbb{E}^{P}\left[X^{+}\right]>0$ and $\mathbb{E}^{P}\left[X^{-}\right]=0$,

Under Assumption 1, we can now take one step further and say that problem LamPr is equivalent to problem LamPL which is stated as:

$$
\begin{array}{ll}
\max & \sum_{n \in \mathscr{N}_{T}} p_{n} x_{n}^{+} \\
\text {s.t. } & \sum_{n \in \mathscr{N}_{T}} p_{n} x_{n}^{-}=1, \\
& Z_{0} \cdot \theta_{0}=0, \\
& Z_{n} \cdot\left(\theta_{n}-\theta_{\pi(n)}\right)=0, \quad \forall n \in \mathscr{N}_{t}, \quad t \geqslant 1, \\
& Z_{n} \cdot \theta_{n}-x_{n}^{+}+x_{n}^{-}=0, \quad \forall n \in \mathscr{N}_{T}, \\
& x_{n}^{+} \geqslant 0, \quad \forall n \in \mathscr{N}_{T}, \\
& x_{n}^{-} \geqslant 0, \quad \forall n \in \mathscr{N}_{T} .
\end{array}
$$

This equivalence can be established using the technique described on p. 151 in [4] as follows. Let us take a solution $\Theta, X^{+}, X^{-}$to LamPr, with $\xi^{-}=\sum_{n \in \mathcal{N}_{T}} p_{n} x_{n}^{-}$. It is easy to see that the point $\frac{1}{\xi^{-}}\left(\Theta, X^{+}, X^{-}\right)$is feasible in LamPL with equal objective function value. For the converse, let $\Psi=\left(\Theta, X^{+}, X^{-}\right)$be a feasible solution to LamPr, and let $\Xi=\left(\bar{\Theta}, \bar{X}^{+}, \bar{X}^{-}\right)$be a feasible solution to LamPL. It is again immediate to see that $\Psi+t \Xi$ is feasible in LamPr for $t \geqslant 0$. Furthermore, we have

$$
\lim _{t \rightarrow \infty} \frac{\mathbb{E}^{P}\left[X^{+}+t \bar{X}^{+}\right]}{\mathbb{E}^{P}\left[X^{-}+t \bar{X}^{-}\right]}=\mathbb{E}^{P}\left[\bar{X}^{+}\right],
$$

which implies that we can find feasible points in LamPr with objective values arbitrarily close to the objective function value at $\Xi$.

We can now construct the linear programming dual of LamPL using Lagrange duality technique which results in the dual linear program (HD1) in variables $y_{n},(n \in \mathscr{N})$ and $V$ :

$$
\begin{array}{ll}
\min & V \\
\text { s.t. } & y_{m} Z_{m}=\sum_{n \in \mathscr{S}(m)} y_{n} Z_{n}, \quad \forall m \in \mathscr{N}_{t}, 0 \leqslant t \leqslant T-1, \\
& p_{n} \leqslant y_{n} \leqslant V p_{n}, \quad \forall n \in \mathscr{N}_{T} .
\end{array}
$$

Ley $Y(V)$ denote the set of $\left\{y_{n}\right\}$ that are feasible in the above problem for a given $V$. Notice that, for $V_{1}<V_{2}$, one has $Y\left(V_{1}\right) \subseteq Y\left(V_{2}\right)$, assuming the respective sets to be non-empty. Hence, the optimal value of $V$ is the minimum value such that the associated set $Y(V)$ is non-empty. 
The dual can also be re-written as (HD2):

$$
\begin{array}{ll}
\min & \max _{n \in \mathscr{N}_{T}} \frac{y_{n}}{p_{n}} \\
\text { s.t. } & y_{m} Z_{m}=\sum_{n \in \mathscr{S}(m)} y_{n} Z_{n}, \quad \forall m \in \mathscr{N}_{t}, 0 \leqslant t \leqslant T-1, \\
& p_{n} \leqslant y_{n}, \quad \forall n \in \mathscr{N}_{T} .
\end{array}
$$

Let $Y$ denote the set of feasible solutions to the above problem. We summarize our findings in the proposition below.

Proposition 2. Under Assumption 1 we have

1. Problem LamP1 is equivalent to problem LamPL.

2. When optimal solutions exist, for any optimal solution $\Theta^{*},\left(X^{+}\right)^{*},\left(X^{-}\right)^{*}, \lambda^{*}$ of LamP1, we have that $\frac{1}{\mathbb{E}^{P}\left[\left(X^{-}\right)^{*}\right]}\left(\Theta^{*},\left(X^{+}\right)^{*},\left(X^{-}\right)^{*}\right)$ is optimal for LamPL.

3. When optimal solutions exist, for any optimal solution $\Theta^{*},\left(X^{+}\right)^{*},\left(X^{-}\right)^{*}$ of LamPL and any $\kappa>0$, we have that $\kappa\left(\Theta^{*},\left(X^{+}\right)^{*},\left(X^{-}\right)^{*}\right), \frac{\mathbb{E}^{P}\left[\left(X^{+}\right)^{*}\right]}{\left.\mathbb{E}^{-}\left(X^{-}\right)^{*}\right]}$ is optimal for LamP1.

4. The supremum $\lambda^{*}$ of $\lambda$ is equal to $\min _{y \in Y} \max _{n \in \mathcal{N}_{T}} \frac{y_{n}}{p_{n}}$.

The last item of the above proposition is essentially the duality result of Bernardo and Ledoit (c.f. Theorem 1 in p. 151 of [2]) which they prove for single-period investments but using an infinite-state setup.

By way of illustration, setting up and solving the problem LamPL for the trinomial numerical example of the previous section, one obtains the largest value of $\lambda$ as six, as the optimal value of the problem LamPL. This is the smallest value of $\lambda$ that does not allow a $\lambda$ gain-loss ratio opportunity. Put in other words, it is the supremum of all values of $\lambda$ allowing a $\lambda$ gain-loss ratio opportunity.

\section{Financing of contingent claims and gain-loss ratio opportunities: positions of writers and buyers}

Now, let us take the viewpoint of a writer of contingent claim $F$ which is generating pay-offs $F_{n},(n>0)$ to the holder (liabilities of the writer), depending on the states $n$ of the market (hence the adjective contingent). The following is a legitimate question on the part of the writer: what is the minimum initial investment needed to replicate the pay-outs $F_{n}$ using securities available in the market with no risk of positive expected terminal wealth falling short of $\lambda$ times the expected negative terminal wealth? King [21] posed a similar question in the context of no-arbitrage pricing, hence for preventing the risk of terminal positions being negative at any state of nature. Here, obviously we are working with an enlarged feasible set of replicating portfolios, if not empty.

Let us now pose the problem of financing of the writer who opts for the $\lambda$ gain-loss ratio opportunity viewpoint rather than the classical arbitrage viewpoint. The writer is facing the stochastic linear programming problem WP1

$$
\begin{array}{ll}
\min & Z_{0} \cdot \theta_{0} \\
\text { s.t. } & Z_{n} \cdot\left(\theta_{n}-\theta_{\pi(n)}\right)=-\beta_{n} F_{n}, \quad \forall n \in \mathscr{N}_{t}, t \geqslant 1 \\
& Z_{n} \cdot \theta_{n}-x_{n}^{+}+x_{n}^{-}=0, \quad \forall n \in \mathscr{N}_{T}, \\
& \sum_{n \in \mathscr{N}_{T}} p_{n} x_{n}^{+}-\lambda \sum_{n \in \mathcal{N}_{T}} p_{n} x_{n}^{-} \geqslant 0, \\
& x_{n}^{+} \geqslant 0, \quad \forall n \in \mathscr{N}_{T}, \\
& x_{n}^{-} \geqslant 0, \quad \forall n \in \mathscr{N}_{T}
\end{array}
$$

as opposed to King's financing problem

$$
\begin{array}{ll}
\min & Z_{0} \cdot \theta_{0} \\
\text { s.t. } & Z_{n} \cdot\left(\theta_{n}-\theta_{\pi(n)}\right)=-\beta_{n} F_{n}, \quad \forall n \in \mathscr{N}_{t}, t \geqslant 1 \\
& Z_{n} \cdot \theta_{n} \geqslant 0, \quad \forall n \in \mathscr{N}_{T} .
\end{array}
$$

Let us assume that a price of $F_{0}$ is attached to a contingent claim $F$. The following definition is useful.

Definition 4. A contingent claim $F$ with price $F_{0}$ is said to be $\lambda$-attainable if there exist vectors $\theta_{n}$ for all $n \in \mathscr{N}$ satisfying:

$$
\begin{aligned}
& Z_{0} \cdot \theta_{0} \leqslant \beta_{0} F_{0}, \\
& Z_{n} \cdot\left(\theta_{n}-\theta_{\pi(n)}\right)=-\beta_{n} F_{n}, \quad \forall n \in \mathscr{N}_{t}, t \geqslant 1
\end{aligned}
$$

and

$$
\mathbb{E}^{P}\left[X^{+}\right]-\lambda \mathbb{E}^{P}\left[X^{-}\right]=0
$$

Proposition 3. At a fixed level $\lambda>1$, assume the discrete vector price process $\left\{Z_{t}\right\}$ does not have a $\lambda$ gain-loss ratio opportunity. Then the minimum initial investment $W_{0}$ required to hedge the claim with no risk of expected positive terminal wealth falling short of $\lambda$ times the expected negative terminal wealth satisfies

$$
W_{0}=\frac{1}{\beta_{0}} \max _{y \in Y(\lambda)} \frac{\sum_{n>0} y_{n} \beta_{n} F_{n}}{y_{0}}
$$

where $Y(\lambda)$ is the set of all $y \in \mathbb{R}^{|\mathcal{H}|}$ satisfying the conditions (3)-(5), i.e., the feasible set of SD1. 
Proof. Let us begin by forming the linear programming dual of problem SP2. Forming the Lagrangian function after attaching multipliers $v_{n},(n>0), w_{n},\left(n \in \mathscr{N}_{T}\right)$ (all unrestricted-in-sign) and $V \geqslant 0$ we obtain

$$
L\left(\Theta, X^{+}, X^{-}, v, w, V\right)=Z_{0} \cdot \theta_{0}+V\left(\lambda \sum_{n \in \mathscr{N}_{T}} p_{n} x_{n}^{-}-\sum_{n \in \mathscr{N}_{T}} p_{n} x_{n}^{+}\right)+\sum_{t=1}^{T} \sum_{n \in \mathscr{N}_{t}} v_{n}\left(Z_{n} \cdot\left(\theta_{n}-\theta_{\pi(n)}\right)+\beta_{N} F_{n}\right)+\sum_{n \in \mathscr{N}_{T}} w_{n}\left(Z_{n} \cdot \theta_{n}-x_{n}^{+}+x_{n}^{-}\right)
$$

that we maximize over the variables $\Theta, X^{+}$, and $X^{-}$separately again. This results in the dual problem WD2.1

$$
\begin{array}{ll}
\max & \sum_{n>0} v_{n} \beta_{n} F_{n} \\
\text { s.t. } & Z_{0}=\sum_{n \in \mathscr{S}(0)} v_{n} Z_{n}, \\
& v_{m} Z_{m}=\sum_{n \in \mathscr{S}(m)} v_{n} Z_{n}, \quad \forall m \in \mathscr{N}_{t}, 1 \leqslant t \leqslant T-1, \\
& V p_{n} \leqslant v_{n} \leqslant V \lambda p_{n}, \quad \forall n \in \mathscr{N}_{T}, \\
& V \geqslant 0 .
\end{array}
$$

We observe that no feasible solution to WD2.1 could have a $V$-component equal to zero as this would lead to infeasibility in the $v$-component. Therefore, it is easy to see that the dual is equivalent to the linear-fractional programming problem (that we refer to as WD2.2) using the equivalences $V=1 / y_{0}$ and $v_{n}=y_{n} / y_{0}$ :

$$
\begin{array}{ll}
\max & \frac{\sum_{n>0} y_{n} \beta_{n} F_{n}}{y_{0}} \\
\text { s.t. } & y_{m} Z_{m}=\sum_{n \in \mathscr{S}(m)} y_{n} Z_{n}, \quad \forall m \in \mathscr{N}_{t}, 0 \leqslant t \leqslant T-1, \\
& p_{n} \leqslant y_{n} \leqslant \lambda p_{n}, \quad \forall n \in \mathscr{N}_{T} .
\end{array}
$$

However, the feasible set of the previous problem is identical to the feasible set $Y(\lambda)$ of the dual SD1 in Proposition 1 . Therefore, if the price process $\left\{Z_{t}\right\}$ does not admit a $\lambda$ gain-loss ratio opportunity, then there exists a feasible solution to the dual SD1, and hence, a feasible solution to the dual problems WD2.2 and WD2.1. Since WD2.1 is feasible and bounded above, the primal problem WP1 is solvable by linear programming duality theory. Hence, the result follows.

Notice that in the previous proof we obtained two equivalent expressions for the dual problem of WP1, namely the dual problem in the statement of the Proposition 3 or WD2.2, which is a linear-fractional programming problem, and the linear programming problem WD2.1 that is used for numerical computation. For future reference, we refer to the feasible set of WD2.1 as $Q(\lambda)$, and to its projection on the set of $v$ 's as $\bar{Q}(\lambda)$. It is not difficult to verify that $\bar{Q}(\lambda)$ is the set of martingale measures $\lambda$-compatible to $P$. Since we observed that no optimal (in fact, feasible) solution to WD2.1 could have a $V$-component equal to zero as this would lead to infeasibility in the $v$-component, by the complementary slackness property of optimal solutions to the primal and the dual problems in linear programming, we should have in all optimal solutions $\left(\Theta, X^{+}, X^{-}\right)$to the primal:

$$
\mathbb{E}^{P}\left[X^{+}\right]-\lambda \mathbb{E}^{P}\left[X^{-}\right]=0
$$

We immediately have the following.

Corollary 1. At fixed level $\lambda>1$, assume the discrete vector price process $\left\{Z_{t}\right\}$ does not allow $\lambda$ gain-loss ratio opportunity. Then, contingent claim $F$ priced at $F_{0}$ is $\lambda$-attainable if and only if

$$
\beta_{0} F_{0} \geqslant \max _{y \in Y(\lambda)} \frac{\sum_{n>0} y_{n} \beta_{n} F_{n}}{y_{0}}
$$

In the light of the above, the minimum acceptable price to the writer of the contingent claim $F$ is given by the expression

$$
F_{0}^{w}=\frac{1}{\beta_{0}} \max _{y \in Y(\lambda)} \frac{\sum_{n>0} y_{n} \beta_{n} F_{n}}{y_{0}} .
$$

Let us now look at the problem from the viewpoint of a potential buyer. The buyer's problem is to decide the maximum price he/she should pay to acquire the claim, with no risk of expected positive terminal wealth falling short of $\lambda$ times the expected negative terminal wealth. This translates into the problem

$$
\begin{array}{ll}
\max & -Z_{0} \cdot \theta_{0} \\
\text { s.t. } & Z_{n} \cdot\left(\theta_{n}-\theta_{\pi(n)}\right)=\beta_{n} F_{n}, \quad \forall n \in \mathscr{N}_{t}, t \geqslant 1, \\
& Z_{n} \cdot \theta_{n}-x_{n}^{+}+x_{n}^{-}=0, \quad \forall n \in \mathscr{N}_{T}, \\
& \sum_{n \in \mathscr{N}_{T}} p_{n} x_{n}^{+}-\lambda \sum_{n \in \mathscr{N}_{T}} p_{n} x_{n}^{-} \geqslant 0, \\
& x_{n}^{+} \geqslant 0, \quad \forall n \in \mathscr{N}_{T}, \\
& x_{n}^{-} \geqslant 0, \quad \forall n \in \mathscr{N}_{T} .
\end{array}
$$

The interpretation of this problem is the following: find the maximum amount needed for acquiring a portfolio replicating the proceeds from the contingent claim without the risk of expected negative wealth magnified by a factor $\lambda$ exceeding the expected positive terminal wealth. By repeating the analysis done for the writer (that we do not reproduce here), we can assert that the maximum acceptable price $F_{0}^{b}$ to the buyer in our framework is given by the following, provided that the price process $\left\{Z_{t}\right\}$ does not admit $\lambda$ gain-loss ratio opportunity (at fixed level $\lambda$ ): 


$$
F_{0}^{b}=\frac{1}{\beta_{0}} \min _{y \in Y(\lambda)} \frac{\sum_{n>0} y_{n} \beta_{n} F_{n}}{y_{0}}
$$

Therefore, for fixed $\lambda>1$ and $P$, we can conclude that the writer's minimum acceptable price and the buyer's maximum acceptable price in a market without $\lambda$ gain-loss ratio opportunity constitute a $\lambda$ gain-loss price interval given as

$$
\left[\frac{1}{\beta_{0}} \min _{y \in Y(\lambda)} \frac{\sum_{n>0} y_{n} \beta_{n} F_{n}}{y_{0}} ; \frac{1}{\beta_{0}} \max _{y \in Y(\lambda)} \frac{\sum_{n>0} y_{n} \beta_{n} F_{n}}{y_{0}}\right] .
$$

We could equally express this interval as

$$
\left[\frac{1}{\beta_{0}} \min _{v, V \in Q(\lambda)} \mathbb{E}^{v}\left[\sum_{t=1}^{T} \beta_{t} F_{t}\right] ; \frac{1}{\beta_{0}} \max _{v, V \in Q(\lambda)} \mathbb{E}^{v}\left[\sum_{t=1}^{T} \beta_{t} F_{t}\right]\right],
$$

where the optimization is over all martingale measures $\lambda$-compatible to $P$. This is the interval of prices which do not induce either the buyer or writer to engage in buying or selling the contingent claim. They can also be thought of as bounds on the price of the contingent claim. Let us recall that the no-arbitrage pricing interval obtained by King [21] corresponds to

$$
\left[\frac{1}{\beta_{0}} \min _{q \in \bar{Q}} \mathbb{E}^{q}\left[\sum_{t=1}^{T} \beta_{t} F_{t}\right] ; \frac{1}{\beta_{0}} \max _{q \in \bar{Q}} \mathbb{E}^{q}\left[\sum_{t=1}^{T} \beta_{t} F_{t}\right]\right] ;
$$

where $\bar{Q}$ is the set of $q \in \mathbb{R}^{|\mathcal{H}|}$ satisfying

$$
\begin{aligned}
& Z_{0}=\sum_{n \in \mathscr{S}(0)} q_{n} Z_{n}, \\
& q_{m} Z_{m}=\sum_{n \in \mathscr{S}(m)} q_{n} Z_{n}, \quad \forall m \in \mathscr{N}_{t}, 1 \leqslant t \leqslant T-1
\end{aligned}
$$

and

$$
q_{n} \geqslant 0, \quad \forall n \in \mathscr{N}_{T}
$$

Clearly, for fixed $\lambda$ we have the inclusion $\bar{Q}(\lambda) \subset \bar{Q}$ using the positivity of $V$. Hence, the pricing interval obtained above is a smaller interval in width in comparison to the arbitrage-free pricing interval of [21]. Notice that the two intervals will become indistinguishable as $\lambda$ tends to infinity. The more interesting question is the behavior of the interval as $\lambda$ is decreased. Before we examine this issue we consider some numerical examples.

Example 2. Consider the same simple market model of Example 1 in Section 3. We assume a contingent claim on the stock, of the European Call type with a strike price equal to 9 is available. Therefore, we have the following pay-off structure: $F_{1}=11, F_{2}=6, F_{3}=0$, corresponding to nodes 1, 2 and 3, respectively. Computing the no-arbitrage bounds using linear programming, one obtains the interval of prices $[2.0 ; 2.2]$ corresponding to the buyer and to the writer's problems, respectively. For $\lambda=8$, the price interval for no $\lambda$ gain-loss ratio opportunity is $[2.09 ; 2.14]$. For $\lambda=7$, the interval becomes $[2.10 ; 2.13]$. Finally, for $\lambda=6$, which is the smallest allowable value for $\lambda$ below which the above derivations lose their validity, the interval shrinks to a single value of 2.125 , since both the buyer and the writer problems return the same optimal value. Therefore, for two investors that are ready to accept an expected gain prospect that is at least six times as large as an expected loss prospect, it is possible to agree on a common price for the contingent claim in question. In this particular example, the problem HD1 for $\lambda^{*}=6$ which is the optimal value for $\lambda$, possesses a single feasible point $y=(2.66,0.33,0.33,2)^{T}$. Dividing the components by 2.66 which is the component $y_{0}$, we obtain the unique equivalent martingale measure $(1 / 8,1 / 8,3 / 4)^{T}$ (which is also $\lambda$ compatible) leading to the unique price of the contingent claim.

Interestingly, the hedging policies of the buyer and the writer at level $\lambda^{*}=6$ need not be identical. For the writer an optimal hedging policy is to short 6.75 units of riskless asset at $t=0$ and buy 0.887 units of the stock. If node 1 were to be reached, the hedging policy dictates to liquidate the position in both the bond and the stock. In case of node 2, the position in the stock is zeroed out, and a position of 0.562 units in the bond is taken. Finally at node 3, the position in the stock is zeroed out, but a short position of 0.094 units remains in the riskless asset. For the buyer an optimal hedging policy is to buy 5.625 units of riskless asset at $t=0$ and short 0.775 units of the stock. At time $t=1$ if node 1 were to be reached, the hedging policy dictates to pass to a position of 1.125 units in the bond, and to a zero position in the stock. In case of node 2 , all positions are zeroed out. At node 3 , the position in the stock is zeroed out while a short position of 0.187 units remains in the riskless asset.

Example 3. Let us now consider a two-period version of the previous example. The market is again described through a trinomial structure. Let the asset price be as in Examples 1 and 2 for time $t=1$. At time $t=2$, from node 1 at which the price is 20 , the price can evolve to 22, 21 and 19 with equal probability, thereby giving the asset price values at nodes $4-6$. From node 2 at which the price takes value equal to 15 , the price can go to 17 or 14 or 13 with equal probability, resulting in the asset price values at nodes $7-9$. Finally, from node 3 , we have as children nodes the node 10 , node 11 and node 12 , with equally likely asset price realizations equal to 9 , 8 and 7 , respectively. Therefore, the trinomial tree contains 9 paths, each with a probability equal to $1 / 9$. The riskless asset is assumed to have value one throughout. It can be verified that this market is arbitrage free.

Solving for the supremum of $\lambda$ values allowing a $\lambda$ gain-loss ratio opportunity, we obtain 14.5 .

Now, let us assume we have a European Call option $F$ on the stock with strike price equal to 14 , resulting in pay-off values $F_{4}=8, F_{5}=7$, $F_{6}=5$ and $F_{7}=3$ where the index corresponds to the node number in the tree (all other values $F_{n}$ are equal to zero). The no-arbitrage bounds yield the interval $[0.33,1.2]$ for this contingent claim. The no- $\lambda$ gain-loss ratio opportunity intervals go as follows: for $\lambda=17$ one has $[0.86 ; 1.00]$, for $\lambda=16,[0.9 ; 0.99]$, for $\lambda=15[0.94 ; 0.98]$. For the limiting value of $\lambda^{*}=14.5$ the bounds again collapse to a single 
price of 0.9718 attained at the same $\lambda$-compatible martingale measure $q_{4}=q_{5}=0.028, q_{6}=0.085, q_{7}=0.042, q_{8}=q_{9}=q_{10}=0.028$, $q_{11}=0.324$ and $q_{12}=0.408$.

Two tables, Tables 1 and 2, summarize the optimal hedge policies of the writer and the buyer, respectively, when the single price is reached. We only report the results for nodes where non-zero portfolio positions are held. The symbols $B$ and $S$ stand for the riskless asset and the stock, respectively. Again, the hedge policies are quite different, but result in an identical price.

Returning to the issue of the behavior of the price interval when $\lambda$ decreases, consider solving the problem LamPL or its dual HD1 (or HD2) for computing the smallest $\lambda$ which does not allow gain-loss ratio opportunities, i.e., $\lambda^{*}$ which is the supremum of values of $\lambda$ yielding a $\lambda$ gain-loss ratio opportunity. If one solves the dual problem HD1 to obtain as optimal solutions $V^{*}, y^{*}$, and if this solution is the unique feasible solution to the linear program HD1, i.e., if the set of equations and inequalities defining the constraints of HD1 for the fixed value of $V^{*}$ admit a unique solution vector $y^{*}$, then this immediately implies that the no- $\lambda$ gain-loss ratio opportunity pricing bounds at level $\lambda=V^{*}$, i.e., the bounds $\frac{1}{\beta_{0}} \min _{y \in Y(\lambda)} \frac{\sum_{n>0} y_{n} \beta_{n} F_{n}}{y_{0}}, \frac{1}{\beta_{0}} \max _{y \in Y(\lambda)} \frac{\sum_{n>0} y_{n} \beta_{n} F_{n}}{y_{0}}$ coincide since both problems possess the common single feasible point $y^{*}$. However, the following example shows that the bounds do not have to coincide for the smallest $\lambda$ value for which there are no $\lambda$ gain-loss ratio opportunities in the market.

Example 4. Let us assume that the market consists of a riskless asset with zero growth rate, and two stocks. The stock price evolves according to a quadrinomial tree with one period as follows. At time $t=0$, the stock price is 10 for both of the stocks. Hence $Z_{0}=\left(\begin{array}{lll}1 & 10 & 10\end{array}\right)^{T}$. At the time $t=1$, the first stock's price can take the values $10,10,15,5$ and the second stock's price can take values 14 , $2,9,11$ with probabilities $0.25,0.2,0.5$ and 0.05 , respectively. Therefore, at node 1 one has $Z_{1}=\left(\begin{array}{lll}1 & 10 & 14\end{array}\right)^{T}$ with $p_{1}=0.25 ;$ at node 2 $Z_{2}=\left(\begin{array}{lll}1 & 10 & 2\end{array}\right)^{T}$ with $p_{2}=0.2$; at node $3 Z_{3}=\left(\begin{array}{lll}1 & 15 & 9\end{array}\right)^{T}$ with $p_{3}=0.5$ and finally at node $4 Z_{4}=\left(\begin{array}{lll}1 & 5 & 11\end{array}\right)^{T}$ with $p_{4}=0.05$. The payoff structure of the contingent claim to be valued is $F_{1}=10, F_{2}=0, F_{3}=0, F_{4}=0$. We find that the minimum $\lambda$ value which does not allow $\lambda$ gain-loss ratio opportunities in the market is 10 . However, for $\lambda=10$, the price interval of the option for no $\lambda$ gain-loss ratio opportunity is $[2.5 ; 5.26]$.

The above example shows that pricing interval does not necessarily reduce to a single point for the smallest $\lambda$. Then, we pose the question for a market in which there is only one bond and one risky asset. Example 5 shows that there is no unique price even under this simple setting.

Example 5. Let us assume that the market consists of a riskless asset with zero growth rate, and a stock. There are 2 periods and the stock price evolves irregularly for both periods. At the first period the tree branches into 2 nodes and at the second period the tree branches into 3 nodes for both of the nodes at $t=1$, i.e., node 1 branches into nodes $3,4,5$ and node 2 branches into nodes $6,7,8$ at period 2 . At time $t=0$, the stock price is 8 . Hence $Z_{0}=\left(\begin{array}{ll}1 & 8\end{array}\right)^{T}$. At the time $t=1$, the stock's price can take the values 5,10 . Therefore, at node 1 one has $Z_{1}=\left(\begin{array}{ll}1 & 5\end{array}\right)^{T}$ and at node $2 Z_{2}=\left(\begin{array}{ll}1 & 10\end{array}\right)^{T}$. At time $t=2$, the stock's price can take the values $2,6,10$ with probabilities $0.2,0.1$ and 0.1 , respectively, given that its price was 5 at time $t=1$ and $13,11,8$ with probabilities $0.05,0.05$ and 0.5 , respectively, given that its price was 10 at time $t=1$. Therefore, at node 3 one has $Z_{3}=\left(\begin{array}{ll}1 & 2\end{array}\right)^{T}$ with $p_{3}=0.2$; at node $4 Z_{4}=\left(\begin{array}{ll}1 & 6\end{array}\right)^{T}$ with $p_{4}=0.1$; at node $5 Z_{5}=\left(\begin{array}{ll}1 & 10\end{array}\right)^{T}$ with $p_{5}=0.1$; at node $6 Z_{6}=\left(\begin{array}{ll}1 & 13\end{array}\right)^{T}$ with $p_{6}=0.05$; at node $7 Z_{7}=\left(\begin{array}{ll}1 & 11\end{array}\right)^{T}$ with $p_{7}=0.05$; and at node $8 Z_{8}=\left(\begin{array}{ll}1 & 8\end{array}\right)^{T}$ with $p_{8}=0.5$. The pay-off structure of the claim to be valued is $F_{3}=3, F_{8}=3$ and 0 elsewhere. We find that the minimum $\lambda$ value which does not allow $\lambda$ gain-loss ratio opportunities in the market is 5 . However, for $\lambda=5$, the price interval of the option for no $\lambda$ gain-loss ratio opportunity is $[1.38 ; 1.56]$.

The natural question at this point is what happens if we work with a simpler setting. The following theorem shows that the martingale measure is unique for the smallest $\lambda$ when there is only a bond and a risky stock in the market with just one period (no intermediary trading is allowed) under a minimal structural assumption on the stochastic scenario tree. The proof is given in the Appendix.

Table 1

The writer's optimal hedge policy for $\lambda=14.5$.

\begin{tabular}{|c|c|c|}
\hline Node & B & $S$ \\
\hline 0 & -4.056 & 0.503 \\
\hline 1 & -14 & 1 \\
\hline 2 & 7.13 & -0.243 \\
\hline 3 & -4.563 & 0.57 \\
\hline 8 & 3.729 & \\
\hline 9 & 3.972 & \\
\hline 10 & 0.57 & \\
\hline 12 & -0.57 & \\
\hline
\end{tabular}

Table 2

The buyer's optimal hedge policy for $\lambda=14.5$.

\begin{tabular}{|c|c|c|}
\hline Node & $B$ & $S$ \\
\hline 0 & -0.915 & -0.006 \\
\hline 1 & -80.465 & 3.972 \\
\hline 2 & 14 & -1 \\
\hline 3 & -15.324 & 1.915 \\
\hline 4 & 14.915 & \\
\hline 5 & 9.944 & \\
\hline 9 & 1 & \\
\hline 10 & 1.915 & \\
\hline 12 & -1.915 & \\
\hline
\end{tabular}


Theorem 3. Assume that there is a bond and a risky stock in the market consisting of one period such that for all $n \in \mathscr{N}_{1}$ (the leaf nodes) $Z_{n}^{1} \neq Z_{\pi(n)}^{1}\left(\right.$ or $\left.Z_{n}^{1} \neq Z_{0}^{1}\right)$. Then, at the smallest value $\lambda^{*}, Y(\lambda)$ is a singleton.

Notice that the analysis of the writer's and buyer's hedging problems can be also be done using a simple utility function and the conjugate duality framework of convex optimization [24]. The utility function corresponding to no-arbitrage is given as

$$
u_{w}(v)=v-I_{v \geqslant 0}(v)
$$

where $I_{v \geqslant 0}$ is the indicator function of convex analysis which equals zero if $v \geqslant 0$, and $+\infty$ otherwise. Our problems involving the gain-loss objective function (and/or constraint) could alternatively be modeled using the equally simple piecewise-linear utility function

$$
u(v)= \begin{cases}v & \text { if } v \geqslant 0 \\ \lambda v & \text { if } v<0\end{cases}
$$

Then, all our results could be obtained using the concave conjugate function $u^{*}$ given by

$$
u^{*}(y)=\inf _{v}(y v-u(v))
$$

which is finite in our case (in fact, zero) provided that $1 \leqslant y \leqslant \lambda$, which are exactly the constraints showing up in our dual problems where the argument of the $u^{*}$ function is precisely $y_{n} / p_{n}$. However, the path taken in the present paper through linear programming duality is simpler and more accessible.

In closing this section we point out that Bernardo and Ledoit's gain-loss ratio results that were obtained in a single-period, non-linear optimization framework are very similar to the approach described above. We showed that similar results can be obtained in a multi-period (finite probability), linear optimization setting, which is simpler yet much more intuitive.

\section{Proportional transaction costs}

The problem of hedging and pricing contingent claims in presence of transaction costs was investigated in e.g. [9,15,17]. In [9], it was assumed that the cost of trading a stock (excluding the numéraire) is proportional to the price. We assume that the proportional transaction costs for buying and selling a stock are different, and there is no transaction cost for the numéraire. An investor who buys one share of stock $j$ when the (discounted with respect to the numéraire) stock price is $Z_{t}^{j}$ pays $Z_{t}^{j}(1+\eta)$ whereas upon selling the investor gets $Z_{t}^{j}(1-\zeta)$, where $\eta$ and $\zeta$ are both in $[0,1)$. Let us now denote the components of $Z_{t}$ corresponding to the indices from 1 to $J$, as the vector $\bar{Z}_{t}$. Similarly, we refer to the components of $Z_{n}$ corresponding to the indices from 1 to $J$, as the vector $\bar{Z}_{n}$, and as $\bar{\theta}_{n}$ to the portfolio positions corresponding to all these stocks excluding the numéraire, for node $n$ of the scenario tree. Then, the arbitrage problem which will be referred as TC1 becomes the following:

$$
\begin{array}{ll}
\max & \sum_{n \in \mathcal{N}_{T}} p_{n} Z_{n} \cdot \theta_{n} \\
\text { s.t. } & \theta_{0}^{0}+\bar{Z}_{0} \cdot \bar{\theta}_{0}+\eta \bar{Z}_{0} \cdot t_{0}^{+}+\zeta \bar{Z}_{0} \cdot t_{0}^{-}=0, \\
& \theta_{n}^{0}-\theta_{\pi(n)}^{0}+\bar{Z}_{n} \cdot\left(\bar{\theta}_{n}-\bar{\theta}_{\pi(n)}\right)+\eta \bar{Z}_{n} \cdot t_{n}^{+}+\zeta \bar{Z}_{n} \cdot t_{n}^{-}=0, \quad \forall n \in \mathscr{N}_{t}, t \geqslant 1, \\
& Z_{n} \cdot \theta_{n} \geqslant 0, \quad \forall n \in \mathscr{N}_{T}, \\
& \bar{\theta}_{0}=t_{0}^{+}-t_{0}^{-}, \\
& \bar{\theta}_{n}-\bar{\theta}_{\pi(n)}=t_{n}^{+}-t_{n}^{-}, \quad \forall n \in \mathscr{N}_{t}, t \geqslant 1, \\
& t_{n}^{+}, t_{n}^{-} \geqslant 0, \quad \forall n \in \mathscr{N}
\end{array}
$$

where $t_{n}^{+}$and $t_{n}^{-}$are vectors in $\mathbb{R}_{+}^{J}$ denoting number of shares bought and sold, respectively at node $n$. The following theorem, which is equivalent to Theorem 4 of [21] states the conditions for no-arbitrage in a market with transaction costs.

Theorem 4. The discrete state stochastic vector process $\left\{Z_{t}\right\}$ is an arbitrage-free market price process if and only if there is at least one probability measure $Q$ equivalent to $P$, which, extended to intermediate nodes recursively as in (1), makes the process $\left\{Z_{t}\right\}$ fulfill the condition

$$
(1-\zeta) \bar{Z}_{t} \leqslant \mathbb{E}^{Q}\left[\bar{Z}_{T} \mid \mathscr{N}_{t}\right] \leqslant(1+\eta) \bar{Z}_{t}, \quad \forall t \leqslant T-1 .
$$

The proof is omitted. It is not hard to see that for $\eta=\zeta=0$ one recovers the statement of Theorem 1 .

The $\lambda$ gain-loss ratio opportunity seeking investor (at a fixed $\lambda$ ) is interested in solving the problem TC2:

$$
\begin{array}{ll}
\max & \sum_{n \in \mathcal{N}_{T}} p_{n} x_{n}^{+}-\lambda \sum_{n \in \mathcal{N}_{T}} p_{n} x_{n}^{-} \\
\text {s.t. } & \theta_{0}^{0}+\bar{Z}_{0} \cdot \bar{\theta}_{0}+\eta \bar{Z}_{0} \cdot t_{0}^{+}+\zeta \bar{Z}_{0} \cdot t_{0}^{-}=0, \\
& \theta_{n}^{0}-\theta_{\pi(n)}^{0}+\bar{Z}_{n} \cdot\left(\bar{\theta}_{n}-\bar{\theta}_{\pi(n)}\right)+\eta \bar{Z}_{n} \cdot t_{n}^{+}+\zeta \bar{Z}_{n} \cdot t_{n}^{-}=0, \quad \forall n \in \mathscr{N}_{t}, t \geqslant 1, \\
& Z_{n} \cdot \theta_{n}-x_{n}^{+}+x_{n}^{-}=0, \quad \forall n \in \mathscr{N}_{T}, \\
& \bar{\theta}_{0}=t_{0}^{+}-t_{0}^{-}, \\
& \bar{\theta}_{n}-\bar{\theta}_{\pi(n)}=t_{n}^{+}-t_{n}^{-}, \quad \forall n \in \mathscr{N}_{t}, t \geqslant 1, \\
& t_{n}^{+}, t_{n}^{-} \geqslant 0, \quad \forall n \in \mathscr{N}, \\
& x_{n}^{+} \geqslant 0, \quad \forall n \in \mathscr{N}_{T}, \\
& x_{n}^{-} \geqslant 0, \quad \forall n \in \mathscr{N}_{T} .
\end{array}
$$

The counterpart of Theorem 2 in this case becomes the following. 
Theorem 5. The discrete state stochastic vector process $\left\{Z_{t}\right\}$ is a $\lambda$ gain-loss ratio opportunity free market price process at level $\lambda$ if and only if there is at least one probability measure $Q \lambda$-compatible to $P$, which, extended to intermediate nodes recursively as in (1), makes the process $\left\{Z_{t}\right\}$ fulfill condition (12).

The proof is relegated to the Appendix. For $\eta=\zeta=0$ one recovers Theorem 2 .

Now, the no-arbitrage price bounds of the previous section are computed by solving

$$
\begin{array}{ll}
\min & \theta_{0}^{0}+\bar{Z}_{0} \cdot \bar{\theta}_{0}+\eta \bar{Z}_{0} \cdot t_{0}^{+}+\zeta \bar{Z}_{0} \cdot t_{0}^{-} \\
\text {s.t. } & \theta_{n}^{0}-\theta_{\pi(n)}^{0}+\bar{Z}_{n} \cdot\left(\bar{\theta}_{n}-\bar{\theta}_{\pi(n)}\right)+\eta \bar{Z}_{n} \cdot t_{n}^{+}+\zeta \bar{Z}_{n} \cdot t_{n}^{-}=-\beta_{n} F_{n}, \quad \forall n \in \mathscr{N}_{t}, t \geqslant 1, \\
& Z_{n} \cdot \theta_{n} \geqslant 0, \quad \forall n \in \mathscr{N}_{T}, \\
& \bar{\theta}_{0}=t_{0}^{+}-t_{0}^{-}, \\
& \bar{\theta}_{n}-\bar{\theta}_{\pi(n)}=t_{n}^{+}-t_{n}^{-}, \quad \forall n \in \mathscr{N}_{t}, t \geqslant 1, \\
& t_{n}^{+}, t_{n}^{-} \geqslant 0, \quad \forall n \in \mathscr{N}
\end{array}
$$

for the writer, and

$$
\begin{array}{ll}
\max & -\theta_{0}^{0}-\bar{Z}_{0} \cdot \bar{\theta}_{0}-\eta \bar{Z}_{0} \cdot t_{0}^{+}-\zeta \bar{Z}_{0} \cdot t_{0}^{-} \\
\text {s.t. } & \theta_{n}^{0}-\theta_{\pi(n)}^{0}+\bar{Z}_{n} \cdot\left(\bar{\theta}_{n}-\bar{\theta}_{\pi(n)}\right)+\eta \bar{Z}_{n} \cdot t_{n}^{+}+\zeta \bar{Z}_{n} \cdot t_{n}^{-}=\beta_{n} F_{n}, \quad \forall n \in \mathscr{N}_{t}, t \geqslant 1, \\
& Z_{n} \cdot \theta_{n} \geqslant 0, \quad \forall n \in \mathscr{N}_{T}, \\
& \bar{\theta}_{0}=t_{0}^{+}-t_{0}^{-}, \\
& \bar{\theta}_{n}-\bar{\theta}_{\pi(n)}=t_{n}^{+}-t_{n}^{-}, \quad \forall n \in \mathscr{N}_{t}, t \geqslant 1, \\
& t_{n}^{+}, t_{n}^{-} \geqslant 0, \quad \forall n \in \mathscr{N}
\end{array}
$$

for the buyer. These bounds are also obtained using the dual expressions:

$$
\left[\frac{1}{\beta_{0}} \min _{q \in \widetilde{Q}(\eta, \zeta)} \mathbb{E}^{Q}\left[\sum_{t=1}^{T} \beta_{t} F_{t}\right] ; \frac{1}{\beta_{0}} \max _{q \in \widetilde{Q}(\eta, \zeta)} \mathbb{E}^{Q}\left[\sum_{t=1}^{T} \beta_{t} F_{t}\right]\right],
$$

where $\widetilde{Q}(\eta, \zeta)$ is the (closure of) set of measures $Q$ equivalent to $P$ such that the process $\left\{\bar{Z}_{t}\right\}$ satisfies condition (12). The proofs are omitted for these results since they are similar to the proof of our next result.

Now, let us consider the no $\lambda$ gain-loss ratio opportunity bounds obtained from the perspective of the buyer and the writer by going through the usual problems in the hedging space:

$$
\begin{array}{ll}
\min & \theta_{0}^{0}+\bar{Z}_{0} \cdot \bar{\theta}_{0}+\eta \bar{Z}_{0} \cdot t_{0}^{+}+\zeta \bar{Z}_{0} \cdot t_{0}^{-} \\
\text {s.t. } & \theta_{n}^{0}-\theta_{\pi(n)}^{0}+\bar{Z}_{n} \cdot\left(\bar{\theta}_{n}-\bar{\theta}_{\pi(n)}\right)+\eta \bar{Z}_{n} \cdot t_{n}^{+}+\zeta \bar{Z}_{n} \cdot t_{n}^{-}=-\beta_{n} F_{n}, \quad \forall n \in \mathscr{N}_{t}, t \geqslant 1, \\
& Z_{n} \cdot \theta_{n}-x_{n}^{+}+x_{n}^{-}=0, \quad \forall n \in \mathscr{N}_{T}, \\
& \sum_{n \in \mathcal{N}_{T}} p_{n} x_{n}^{+}-\lambda \sum_{n \in \mathscr{N}_{T}} p_{n} x_{n}^{-} \geqslant 0, \\
& \bar{\theta}_{0}=t_{0}^{+}-t_{0}^{-}, \\
& \bar{\theta}_{n}-\bar{\theta}_{\pi(n)}=t_{n}^{+}-t_{n}^{-}, \quad \forall n \in \mathscr{N}_{t}, t \geqslant 1, \\
& t_{n}^{+}, t_{n}^{-} \geqslant 0, \quad \forall n \in \mathscr{N}, \\
& x_{n}^{+} \geqslant 0, \quad \forall n \in \mathscr{N}_{T}, \\
& x_{n}^{-} \geqslant 0, \quad \forall n \in \mathscr{N}_{T}
\end{array}
$$

for the writer, and

$$
\begin{array}{ll}
\max & -\theta_{0}^{0}-\bar{Z}_{0} \cdot \bar{\theta}_{0}-\eta \bar{Z}_{0} \cdot t_{0}^{+}-\zeta \bar{Z}_{0} \cdot t_{0}^{-} \\
\text {s.t. } & \theta_{n}^{0}-\theta_{\pi(n)}^{0}+\bar{Z}_{n} \cdot\left(\bar{\theta}_{n}-\bar{\theta}_{\pi(n)}\right)+\eta \bar{Z}_{n} \cdot t_{n}^{+}+\zeta \bar{Z}_{n} \cdot t_{n}^{-}=\beta_{n} F_{n}, \quad \forall n \in \mathscr{N}_{t}, t \geqslant 1, \\
& Z_{n} \cdot \theta_{n}-x_{n}^{+}+x_{n}^{-}=0, \quad \forall n \in \mathscr{N}_{T}, \\
& \sum_{n \in \mathcal{N}_{T}} p_{n} x_{n}^{+}-\lambda \sum_{n \in \mathcal{N}_{T}} p_{n} x_{n}^{-} \geqslant 0, \\
& \bar{\theta}_{0}=t_{0}^{+}-t_{0}^{-}, \\
& \bar{\theta}_{n}-\bar{\theta}_{\pi(n)}=t_{n}^{+}-t_{n}^{-}, \quad \forall n \in \mathscr{N}_{t}, t \geqslant 1, \\
& t_{n}^{+}, t_{n}^{-} \geqslant 0, \quad \forall n \in \mathscr{N}, \\
& x_{n}^{+} \geqslant 0, \quad \forall n \in \mathscr{N}_{T}, \\
& x_{n}^{-} \geqslant 0, \quad \forall n \in \mathscr{N}_{T}
\end{array}
$$

for the buyer. We see that the fourth and the fifth constraints can be used to get rid of variables $\bar{\theta}$ in the formulation of the above problem. Since $\bar{\theta}_{n}-\bar{\theta}_{\pi(n)}=t_{n}^{+}-t_{n}^{-}, \forall n \in \mathscr{N}_{t}, t \geqslant 1$ and $\bar{\theta}_{0}=t_{0}^{+}-t_{0}^{-}$, it becomes $\bar{\theta}_{n}=t_{n}^{+}-t_{n}^{-}+t_{0}^{+}-t_{0}^{-}, \forall n \in \mathscr{N}_{1}$. Using the same reasoning we have $\bar{\theta}_{n}=\sum_{m \in A(n)}\left(t_{m}^{+}-t_{m}^{-}\right), \forall n \in \mathcal{N}$. Then we obtain the following linear program for the writer: 


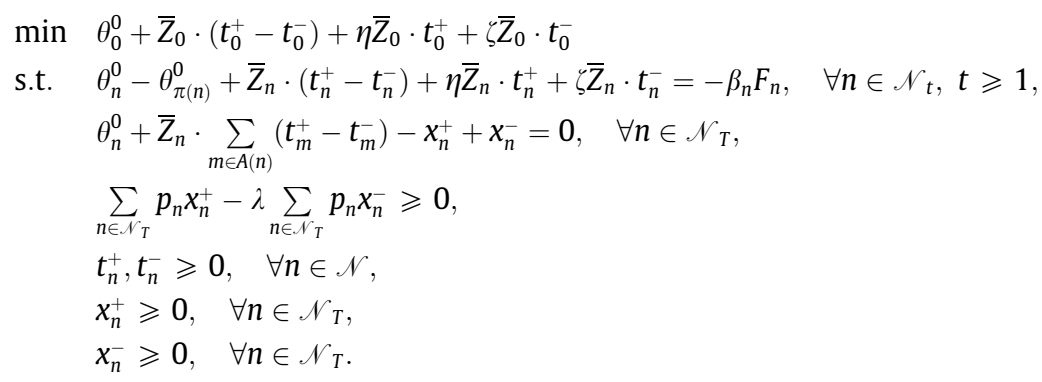

The dual problem of this program is

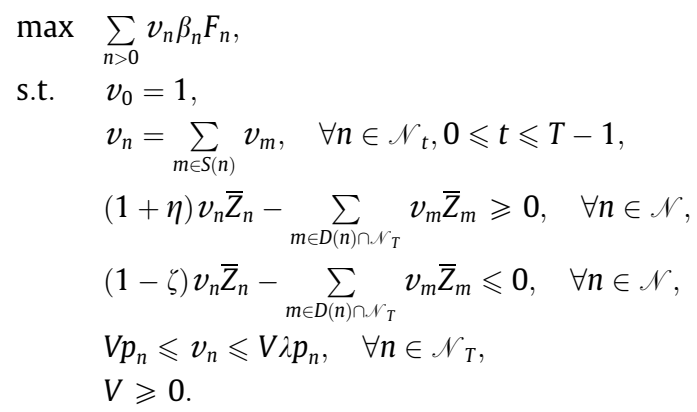

Denote the feasible set of the above dual problem bt $\widetilde{\mathscr{Q}}(\lambda, \eta, \zeta)$, i.e., the set of probability measures $v_{n}$ and positive $V$ such that

$$
(1-\zeta) \bar{Z}_{t} \leqslant \mathbb{E}^{v}\left[\bar{Z}_{T} \mid \mathscr{N}_{t}\right] \leqslant(1+\eta) \bar{Z}_{t}, \quad \forall t \leqslant T-1
$$

and $V p_{n} \leqslant v_{n} \leqslant V \lambda p_{n}, \forall n \in \mathscr{N}_{T}$.

By setting $y_{0}=1 / V$ and $y_{n}=v_{n} / V$, and simplifying we obtain the following equivalent program:

$$
\begin{array}{ll}
\max & \frac{\sum_{n>0} y_{n} \beta_{n} F_{n}}{y_{0}}, \\
\text { s.t. } & y_{n}=\sum_{m \in C(n)} y_{m}, \quad \forall n \in \mathscr{N}_{t}, 0 \leqslant t \leqslant T-1, \\
& (1+\eta) y_{n} \bar{Z}_{n}-\sum_{m \in D(n) \cap \mathcal{N}_{T}} y_{m} \bar{Z}_{m} \geqslant 0, \quad \forall n \in \mathscr{N}, \\
& (1-\zeta) y_{n} \bar{Z}_{n}-\sum_{m \in D(n) \cap \mathcal{N}_{T}} y_{m} \bar{Z}_{m} \leqslant 0, \quad \forall n \in \mathscr{N}, \\
& p_{n} \leqslant y_{n} \leqslant \lambda p_{n}, \quad \forall n \in \mathscr{N}_{T} .
\end{array}
$$

Denote the feasible set of the previous problem $\tilde{\mathscr{Y}}(\lambda, \eta, \zeta)$. Going through a similar derivation for the buyer's case (omitted for brevity) we have proved the following result.

Proposition 4. The price interval of a contingent claim for no $\lambda$ gain-loss ratio opportunity at level $\lambda$ is

$$
\left[\frac{1}{\beta_{0}} \min _{q, V \in \widetilde{\mathscr{Q}}(\lambda, \eta, \zeta)} \mathbb{E}^{Q}\left[\sum_{t=1}^{T} \beta_{t} F_{t}\right] ; \frac{1}{\beta_{0}} \max _{q, V \in \widetilde{\mathscr{Q}}(\lambda, \eta, \zeta)} \mathbb{E}^{Q}\left[\sum_{t=1}^{T} \beta_{t} F_{t}\right]\right]
$$

or, equivalently

$$
\left[\frac{1}{\beta_{0}} \min _{y \in \mathscr{\mathscr { Y }}(\lambda, \eta, \zeta)} \frac{\sum_{n>0} y_{n} \beta_{n} F_{n}}{y_{0}} ; \frac{1}{\beta_{0}} \max _{y \in \widetilde{\mathscr{Y}}(\lambda, \eta, \zeta)} \frac{\sum_{n>0} y_{n} \beta_{n} F_{n}}{y_{0}}\right] .
$$

Obviously, the no $\lambda$ gain-loss ratio opportunity bounds are tighter compared to the no-arbitrage bounds. Notice that $\widetilde{\mathscr{Q}}(\lambda, 0,0)$ and $\widetilde{\mathscr{Y}}(\lambda, 0,0)$ coincide with $Q(\lambda)$ and $Y(\lambda)$, respectively.

Example 6. Considering the same problem as in Example 2 with $\eta=\zeta=0.1$, the supremum of the values of $\lambda$ allowing a $\lambda$ gain-loss ratio opportunity opportunity is computed to 3.715 (notice the drop from 6 in the case of no transaction costs). The no-arbitrage interval for the contingent claim is found to be $[1.2 ; 3.08]$. At $\lambda=4$, the no $\lambda$ gain-loss ratio opportunity interval is $[2.83 ; 2.98]$. At $\lambda=3.715$ which is the limiting value, the common bound is equal to 2.97 . The unique measure leading to this common price is given as $q_{1}=q_{2}=0.175$ and $q_{3}=0.65$.

\section{Conclusions}

We studied the problem of pricing and hedging contingent claims in incomplete markets in a multi-period linear optimization (discrete time, finite probability space) framework. We developed an extension of the concept of no-arbitrage pricing ( $\lambda$ gain-loss ratio 
opportunity) based on expected positive and negative final wealth positions, which allow to obtain arbitrage only in the limit as a gain-loss preference parameter tends to infinity. We analyzed the resulting optimization problems using linear programming duality. We showed that the pricing bounds obtained from our analysis are tighter than the no-arbitrage pricing bounds. This result, in line with the Bernardo and Ledoit [2] single-period results, was also obtained for a multi-period model in the computationally more tractable linear programming environment. Our results indicated that for a limiting value of risk aversion parameter that can be computed easily, a unique price for a contingent claim in incomplete markets may be found (although this is not guaranteed) while different hedging schemes exist for different sides of the same trade. We also extended our results to markets with transaction costs.

\section{Appendix A}

Proof (Proof of Proposition 1). We should first note that the assumption of the existence of a $\lambda$ gain-loss ratio opportunity implies that LamP1 and LamPr have both non-empty feasible sets. We can see this fact by the problem SP1 and the definition of a $\lambda$ gain-loss ratio opportunity (see problem SP1 and the paragraph following it) based on SP1. Assume that the optimal value of LamP1 is the finite number $\bar{\lambda}$ and the optimal value of LamPr is greater than $\bar{\lambda}$. Then, problem LamPr must have a feasible solution $\Theta, X^{+}, X^{-}$which has an objective value $\lambda^{\prime}$ that is greater than $\bar{\lambda}$ by the definition of a supremum. Then we see that $\Theta, X^{+}, X^{-}, \lambda^{\prime}-\epsilon$ with $\epsilon<\lambda^{\prime}-\bar{\lambda}$ constitute another feasible solution to LamP1 with the objective value $\lambda^{\prime}-\epsilon$. But, this contradicts with the assumption that $\bar{\lambda}$ is the optimal value of LamP1 since $\lambda^{\prime}-\epsilon>\bar{\lambda}$. Hence, if LamP1 has a finite optimal value, LamPr cannot have an optimal value greater than that. Conversely, assume that the optimal value of LamPr is the finite number $\bar{\lambda}$ and the optimal value of LamP1 is greater than that. Then, LamP1 must have a feasible solution $\Theta, X^{+}, X^{-}, \lambda^{\prime}$ which has an objective value $\lambda^{\prime}$ that is greater than $\bar{\lambda}$. Then, $\Theta, X^{+}, X^{-}$constitute another feasible solution to LamPr with the objective value greater than $\lambda^{\prime}$ thus greater than $\bar{\lambda}$. Again, this contradicts with our assumption that $\bar{\lambda}$ is the optimal value of LamPr. Hence, if LamPr has a finite optimal value, LamP1 cannot have an optimal value greater than that. Using these facts we conclude that, if one of the problems has a finite optimal value the other one also has the same optimal value and if one of them is unbounded, the other one is also unbounded. It proves that they are equivalent when there is a $\lambda$ gain-loss ratio opportunity.

Proof (Proof of Theorem 3). Let $L=\left|\mathscr{N}_{1}\right|$ be the number of leaf nodes. Let us view the problem of computing the smallest $\lambda$ such that $Y(\lambda)$ has a solution, as a parametric feasibility problem with parameter $\lambda$. That is, for fixed $\lambda \geqslant 1$ we are interested to determine whether the restriction $A^{L}$ onto the $L$-dimensional space composed of $y_{n}$ for all $n \in \mathscr{N}_{1}$ (i.e., $\mathbb{R}^{L}$ ) of the set $A=\left\{y_{n}: y_{0} Z_{0}=\sum_{n \in \mathscr{S}(0)} y_{n} Z_{n}\right\}$, has non-empty intersection with the $L$-dimensional box $H_{\lambda}=\left\{y_{n}: p_{n} \leqslant y_{n} \leqslant \lambda p_{n}, \forall n \in \mathscr{N}_{1}.\right\}$.

Notice that $A^{L}$ defines an affine set in the $L$-dimensional space of "leaf variables".

If the smallest value $\lambda^{*}$ of $\lambda$, such that $A^{L} \cap H_{\lambda}$ is not empty, is equal to one, the theorem clearly holds because the set of solutions is necessarily a singleton in this case. So, we assume $\lambda^{*}>1$. Let us fix some $\lambda>1$ such that $A^{L} \cap H_{\lambda}$ is non-empty and is not a singleton. There are two cases to consider.

Case 1 There exist two "distinct", meaning all components different, $L$-vectors, $y^{1}$ and $y^{2}$ say, in $A^{L} \cap H_{\lambda}$. In this case, $\lambda$ can be reduced since $A^{L} \cap H_{\lambda}$ is a convex set and any convex combination of $y^{1}$ and $y^{2}$ is also in the set.

Case 2 There are no "distinct" $L$-vectors $y^{1}$ and $y^{2}$ say, in $A^{L} \cap H_{\lambda}$. For this case, we first observe that there must be $i \in \mathscr{N}_{1}$ such that $y_{i}^{1}=y_{i}^{2}, \forall y^{1}, y^{2} \in A^{L} \cap H_{\lambda}$. Otherwise, we would be able to find a set of vectors $\left\{y^{1}, y^{2}, \ldots, y^{k}: \nexists i \in \mathscr{N}_{1}, y_{i}^{a}=y_{i}^{b}, \forall a, b \in\{1, \ldots, k\}\right\}$. Then, we could take a convex combination of these vectors in $A^{L} \cap H_{\lambda}$, which is a distinct vector with $\left\{y^{1}, y^{2}, \ldots, y^{k}\right\}$. This contradicts with the assumption of case 2 . Our second observation is there must be $i \in \mathscr{N}_{1}$ such that $y_{i}=p_{i}, \forall y \in A^{L} \cap H_{\lambda}$. Otherwise, we would find a set of vectors $\left\{y^{1}, y^{2}, \ldots, y^{k}: \nexists i \in \mathscr{N}_{1}, y_{i}^{a}=p_{i}, \forall a \in\{1, \ldots, k\}\right\}$ and we could get a convex combination of these vectors $y^{\prime}$ such that $\nexists i \in \mathscr{N}_{1}, y_{i}^{\prime}=p_{i}$. One can see that $\bar{y}: \bar{y}_{i}=0, \forall \in \mathscr{N}_{1}$ is a feasible solution to the equations defining the set $A$. Then, we could take a convex combination of $y^{\prime}$ and $\bar{y}$ which is distinct with $y^{\prime}$ and which is in $A^{L} \cap H_{\lambda}$, contradicting the assumption of case 2 . After these two observations we need to analyze the system of equations defining the set $A$. For a risky asset and a bond there are just two equations. The first one is $y_{0}=\sum_{n \in \mathscr{S}(0)} y_{n}$. The second one is $y_{0} Z_{0}^{1}=\sum_{n \in \mathscr{S}(0)} y_{n} Z_{n}^{1}$. A solution of these two equations satisfies $\sum_{n \in \mathscr{S}(0)} y_{n}\left(Z_{n}^{1}-Z_{0}^{1}\right)=0$. Let $\alpha_{n}=\left(Z_{n}^{1}-Z_{0}^{1}\right) ; \forall n \in \mathscr{N}_{1}$. Note that our structural assumption implies that $\alpha_{n} \neq 0 ; \forall n \in \mathscr{N}_{1}$. Let us say that $i \in \mathscr{N}_{1}$ is such that $y_{i}=p_{i}, \forall y \in A^{L} \cap H_{\lambda}$ and $y$ be any vector in $A^{L} \cap H_{\lambda}$. First assume that $\alpha_{i}>0$. Consider any $j \in \mathscr{N}_{1}$. If $\alpha_{j}>0$ then $y_{j}=p_{j}$. Otherwise, we could find $\epsilon$ small enough such that when we decrease $y_{j}$ by $\epsilon$ and increase $y_{i}$ by $\alpha_{j} \epsilon / \alpha_{i}$ resulting in another solution in $A^{L} \cap H_{\lambda}$ with $y_{i} \neq p_{i}$, which is a contradiction. Conversely, if $\alpha_{j}<0$ then $y_{j}=\lambda p_{j}$. Otherwise, we could find $\epsilon$ small enough such that increasing $y_{j}$ by $\epsilon$ and increasing $y_{i}$ by $-\alpha_{j} \epsilon / \alpha_{i}$ we could get another solution in $A^{L} \cap H_{\lambda}$ with $y_{i} \neq p_{i}$ which is again a contradiction. A similar argument follows for the case $\alpha_{i}<0$. Therefore there can only be a unique solution for this case contradicting with the assumption $A^{L} \cap H_{\lambda}$ is not a singleton.

Therefore, Case 2 cannot occur, i.e., we are always in Case 1 i.e., $\lambda$ can be reduced, if $A^{L} \cap H_{\lambda}$ is not a singleton.

A consequence of the above reasoning is that if $\lambda$ cannot be reduced, i.e., $\lambda=\lambda^{*}$, then $A^{L} \cap H_{\lambda}$ must be a singleton.

Proof (Proof of Theorem 5). We prove the necessity part first. Assume that the market has no $\lambda$ gain-loss ratio opportunity. We see that the fourth and the fifth constraints can be used to get rid of variables $\bar{\theta}$ in the formulation of TC2. Since $\bar{\theta}_{n}-\bar{\theta}_{\pi(n)}=t_{n}^{+}-t_{n}^{-}, \forall n \in \mathscr{N}_{t}, t \geqslant 1$ and $\bar{\theta}_{0}=t_{0}^{+}-t_{0}^{-}$, it becomes $\bar{\theta}_{n}=t_{n}^{+}-t_{n}^{-}+t_{0}^{+}-t_{0}^{-}, \forall n \in \mathscr{N}_{1}$. Using the same reasoning we have $\bar{\theta}_{n}=\sum_{m \in A(n)}\left(t_{m}^{+}-t_{m}^{-}\right), \forall n \in \mathscr{N}$. Then TC2 becomes: 


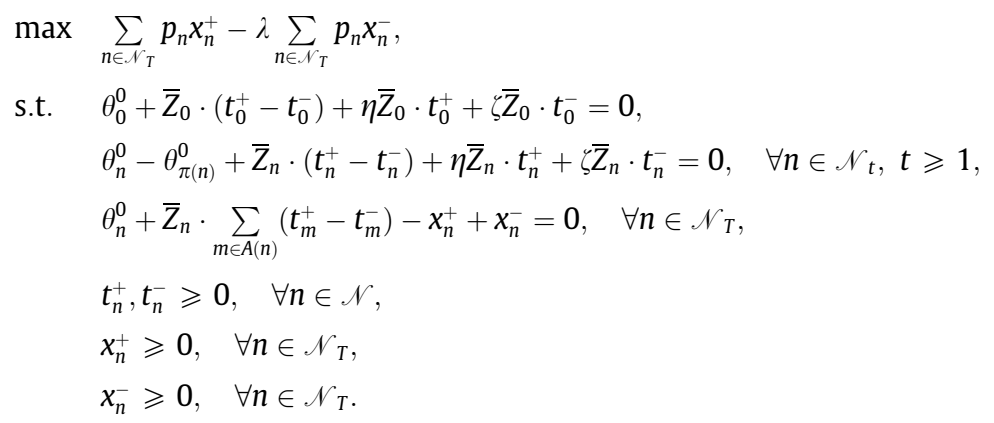

The dual of this problem is the following feasibility problem:

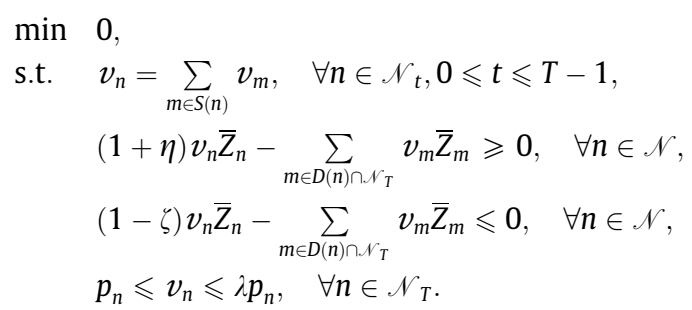

If there is no $\lambda$ gain-loss ratio opportunity, the optimal value of TC2 is equal to zero. Therefore, by linear programming duality, the dual problem is also solvable (in fact, feasible since the dual is only a feasibility problem). Let us take any feasible solution $v_{n},(n \in \mathscr{N})$ of the dual problem. Since $v_{n} \geqslant p_{n}$, it follows that $v_{n}$ is a strictly positive process such that the sum of $v_{n}$ over all states $n \in \mathscr{N}_{t}$ in each time period $t$ sums to $v_{0}$. Now, define the process $q_{n}=v_{n} / v_{0}$, for each $n \in \mathscr{N}$. Obviously, this defines a probability measure $Q$ over the leaf (terminal) nodes $n \in \mathscr{N}_{T}$ and it extends to intermediate nodes recursively as in (1) as an implication of the first constraint in the dual problem. Furthermore, we can rewrite the second and the third constraints of the dual problem with the newly defined weights $q_{n}$ as

$$
\begin{aligned}
& (1+\eta) q_{n} \bar{Z}_{n}-\sum_{m \in D(n) \cap \mathscr{N}_{T}} q_{m} \bar{Z}_{m} \geqslant 0, \quad \forall n \in \mathscr{N}, \\
& (1-\zeta) q_{n} \bar{Z}_{n}-\sum_{m \in D(n) \cap \mathcal{N}_{T}} q_{m} \bar{Z}_{m} \leqslant 0, \quad \forall n \in \mathscr{N},
\end{aligned}
$$

with $q_{0}=1$, and all $q_{n}>0$. Therefore, by constructing the probability measure $Q$ we have constructed an equivalent measure which makes the process $\left\{Z_{t}\right\}$ fulfill condition (12). By definition of the measure $q_{n}$, we have using the last constraint of the dual problem

$$
p_{n} \leqslant q_{n} v_{0} \leqslant \lambda p_{n}, \quad \forall n \in \mathscr{N}_{T}
$$

or equivalently,

$$
p_{n} / q_{n} \leqslant v_{0} \leqslant \lambda p_{n} / q_{n} \quad \forall n \in \mathscr{N}_{T}
$$

which implies that $q_{n}, n \in \mathscr{N}_{T}$ constitute a measure $\lambda$-compatible to $P$. This concludes the necessity part.

Suppose $Q$ is a probability measure $\lambda$-compatible to $P$, which extends to intermediate nodes recursively as in (1) and which makes the process $\left\{Z_{t}\right\}$ fulfill condition (12). Therefore, we have

$$
\begin{aligned}
& (1+\eta) q_{n} \bar{Z}_{n}-\sum_{m \in D(n) \cap \mathscr{N}_{T}} q_{m} \bar{Z}_{m} \geqslant 0, \quad \forall n \in \mathscr{N}, \\
& (1-\zeta) q_{n} \bar{Z}_{n}-\sum_{m \in D(n) \cap \mathscr{N}_{T}} q_{m} \bar{Z}_{m} \leqslant 0, \quad \forall n \in \mathscr{N},
\end{aligned}
$$

with $q_{0}=1$, and all $q_{n}>0$, while the condition $\max _{n \in \mathcal{N}_{T}} p_{n} / q_{n} \leqslant \lambda \min _{n \in \mathcal{N}_{T}} p_{n} / q_{n}$ holds. If the previous inequality holds as an equality, choose the right-hand (or, the left-hand) of the inequality as a factor $v_{0}$ and set $v_{n}=q_{n} v_{0}$ for all $n \in \mathscr{N}$. If the inequality is not tight, any value $v_{0}$ in the interval $\left[\max _{n \in \mathcal{N}_{T}} p_{n} / q_{n}, \lambda \min _{n \in \mathcal{N}_{T}} p_{n} / q_{n}\right.$ ] can be chosen. It is easily verified that $v_{n}, n \in \mathscr{N}$ so defined satisfy the constraints of the dual problem. Since the dual problem is feasible, the primal TC2 is bounded above (in fact, its optimal value is zero) and no $\lambda$ gain-loss ratio opportunity exists in the system.

\section{References}

[1] N. Barberis, M. Huand, T. Santos, Prospect theory and asset prices, Quarterly Journal of Economics CXVI (1) (2001) 1-53.

[2] A.E. Bernardo, O. Ledoit, Gain, loss and asset pricing, Journal of Political Economy 108 (2000) 144-172.

[3] J.R. Birge, F. Louveaux, Introduction to Stochastic Programming, Springer Series in Operations Research, Springer, New York, 1997.

[4] S. Boyd, L. Vandenberghe, Convex Optimization, Cambridge University Press, Cambridge, 2004.

[5] P. Carr, H. Geman, D.B. Madan, Pricing and hedging in incomplete markets, Journal of Financial Economics 62 (2001) $131-167$.

[6] A. Cerny, S.D. Hodges, The theory of good-deal pricing in financial markets, in: Mathematical Finance - Bachelier Congress 2000 , Springer-Verlag, Berlin, 2000.

[7] A. Cerny, Generalized Sharpe ratios and asset pricing in incomplete markets, European Finance Review 7 (2003) 191-233.

[8] J.H. Cochrane, J. Saa-Requejo, Beyond arbitrage: Good-deal asset price bounds in incomplete markets, Journal of Political Economy 108 (2000) 79-119.

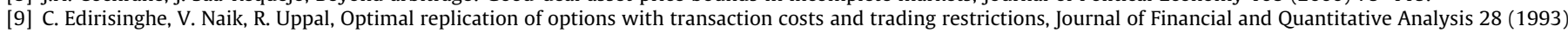
117-138. 
[10] H. Föllmer, A. Schied, Stochastic Finance: An Introduction in Discrete Time, De Gruyter Studies in Mathematics, vol. 27, second ed., Berlin, 2004.

[11] S.D. Flåm, Option pricing by mathematical programming, Optimization 57 (2008) 165-182.

[12] L. Grüne, W. Semmler, Asset pricing with loss aversion, Journal of Economic Dynamics and Control 32 (2008) 3253-3274.

[13] J.M. Harrison, D.M. Kreps, Martingales and arbitrage in multi-period securities markets, Journal of Economic Theory 20 (1979) $381-408$

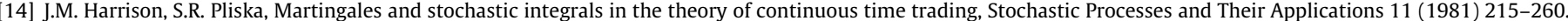

[15] S.D. Hodges, A. Neuberger, Optimal replication of contingent claims under transaction costs, Review of Futures Markets 8 (1989) $222-239$.

[16] C.F. Huang, R.H. Litzenberger, Foundations for Financial Economics, North-Holland, Amsterdam, 1988

[17] E. Jouini, H. Kallal, Martingales and arbitrage in securities markets with transaction costs, Journal of Economic Theory 66 (1995) $178-197$.

[18] P. Judice, Foundations and Applications of Good-Deal Pricing I: Single-Period Market Models, Working Paper, May 2005.

[19] D. Kahneman, A. Tversky, Prospect theory: An analysis of decision under risk, Econometrica 47 (1979) $263-291$.

[20] J. Kallsen, Utility-based Derivative Pricing in Incomplete Markets, in: Mathematical Finance - Bachelier Congress 2000 , Springer-Verlag, Berlin, 2000.

21] A.J. King, Duality and martingales: A stochastic programming perspective on contingent claims, Mathematical Programming Series B 91 (2002) 543-562.

[22] I.R. Longarela, A simple linear programming approach to gain, loss and asset pricing, Topics in Theoretical Economics 2 (1) (2002). Article 4.

23] S. Pliska, Introduction to Mathematical Finance: Discrete Time Models, Blackwell Publishers, Maldon, 2001.

$24]$ T.R. Rockafellar, Convex Analysis, Princeton University Press, Princeton, New Jersey, 1970.

[25] B. Roorda, J.M. Schumacher, J. Engwerda, Coherent acceptability measures in multiperiod models, Mathematical Finance 15 (2005) 589-612.

[26] S. Ross, The arbitrage theory of capital asset pricing, Journal of Economic Theory 13 (1976) 341-360.

[27] S. Ross, A simple approach to the valuation of risky streams, Journal of Business 51 (1978) 453-475.

[28] J. Staum, Fundamental theorems of asset pricing for good deal bounds, Mathematical Finance 14 (2004) 141-161.

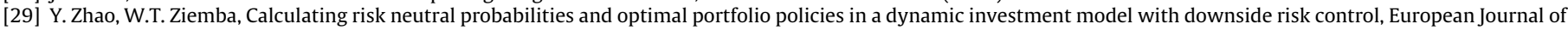
Operational Research 185 (2008) 1525-1540. 